This item was submitted to Loughborough's Research Repository by the author.

Items in Figshare are protected by copyright, with all rights reserved, unless otherwise indicated.

\title{
Dynamical mechanism for sharp orientation tuning in an integrate-and-fire model of a cortical hypercolumn.
}

PLEASE CITE THE PUBLISHED VERSION

LICENCE

CC BY-NC-ND 4.0

\section{REPOSITORY RECORD}

Bressloff, P.C., N.W. Bressloff, and J.D. Cowan. 2019. "Dynamical Mechanism for Sharp Orientation Tuning in an Integrate-and-fire Model of a Cortical Hypercolumn.”. figshare. https://hdl.handle.net/2134/825. 


\title{
Dynamical mechanism for sharp orientation tuning in an integrate-and-fire model of a cortical hypercolumn
}

\author{
P. C. Bressloff \\ Nonlinear and Complex Systems Group \\ Department of Mathematical Sciences, Loughborough University \\ Loughborough, Leicestershire LE11 3TU, U. K. \\ N. W. Bressloff \\ Computational and Engineering Design Centre \\ Department of Aeronautics and Astronautics, University of Southampton \\ Southampton, SO17 1BJ, U.K. \\ J. D. Cowan \\ Department of Mathematics, University of Chicago \\ Illinois, 60637, U. S. A.
}

April 19, 1999

\begin{abstract}
Orientation tuning in a ring of pulse-coupled integrate-and-fire (IF) neurons is analyzed in terms of spontaneous pattern formation. It is shown how the ring bifurcates from a synchronous state to a non-phase-locked state whose spike trains are characterized by clustered but irregular fluctuations of the inter-spike intervals (ISIs). The separation of these clusters in phase space results in a localized peak of activity as measured by the time-averaged firing rate of the neurons. This generates a sharp orientation tuning curve that can lock to a slowly rotating, weakly tuned external stimulus. Under certain conditions the peak can slowly rotate even to a fixed external stimulus. The ring also exhibits hysteresis due to the
\end{abstract}


subcritical nature of the bifurcation to sharp orientation tuning. Such behavior is shown to be consistent with a corresponding analog version of the IF model in the limit of slow synpatic interactions. For fast synapses, the deterministic fluctuations of the ISIs associated with the tuning curve can support a coefficient of variation $(\mathrm{CV})$ of order unity.

\section{Introduction}

Recent studies of the formation of localized spatial patterns in one- and two-dimensional neural networks have been used to investigate a variety of neuronal processes including orientation selectivity in primary visual cortex (Ben-Yishai, Lev and Sompolinsky 1995, 1997; Hansel and Sompolinsky 1997, Mundel, Dimitrov and Cowan 1997), the coding of arm movements in motor cortex (Lukashin and Georgopolous 1994; Georgopolous 1995), and the control of saccadic eye movements (Zhang 1996). The networks considered in these studies are based on a simplified rate or analog model of a neuron, in which the state of each neuron is characterized by a single continuous variable that determines its short-term average output activity (Cowan 1968, Wilson and Cowan 1972). All of the above models involve the same basic dynamical mechanism for the formation of localized patterns, namely, spontaneous symmetry breaking from a uniform resting state (Cowan 1982). Localized structures consisting of a single peak of high activity occur when the maximum (spatial) Fourier component of the combination of excitatory and inhibitory interactions between neurons has a wavelength comparable to the size of the network. Typically, such networks have periodic boundary conditions so that unravelling the network results in a spatially periodic pattern of activity, as studied previously by Ermentrout and Cowan (1979a,b); see also the review by Ermentrout (1998).

In contrast to mean firing rate models, there has been relatively little analytical work on pattern formation in more realistic spiking models. A number of numerical studies have shown that localized activity profiles can occur in networks of Hodgkin-Huxley neurons (Lukashin and Georgopolous 1994; Hansel and Sompolinsky 1996). Moreover, both local (Somers, Nelson and Sur 1995) and global (Usher, Stemmler, Koch and Olami 1994) patterns of activity have been found in integrate-and-fire (IF) networks. Recently, a dynamical theory of global pattern formation in IF networks has been developed in terms of the nonlinear map of the neuronal firing 
times (Bressloff and Coombes 1998b,1999). A linear stability analysis of this map shows how, in the case of short-range excitation and long-range inhibition, a network that is synchronized in the weak coupling regime can destabilize as the strength of coupling is increased leading to a state characterized by clustered but irregular fluctuations of the inter-spike intervals (ISIs). The separation of these clusters in phase space results in a spatially periodic pattern of mean (time-averaged) firing rate across the network, which is modulated by deterministic fluctuations in the instantaneous firing rates.

In this paper we apply the theory of Bressloff and Coombes $(1998 \mathrm{~b}, 1999)$ to the analysis of localized pattern formation in an integrate-and-fire version of the model of sharp orientation tuning developed by Hansel and Sompolinsky (1997) and Ben-Yishai et al (1995, 1997). We first study a corresponding analog model in which the outputs of the neurons are taken to be mean firing rates. Since the resulting firing rate function is nonlinear, rather than semilinear, it is not possible to construct exact solutions for the orientation tuning curves along the lines of Hansel and Sompolinsky (1997). Instead, we investigate the existence and stability of such activity profiles using bifurcation theory. We show how a uniform resting state destabilizes to a stable localized pattern as the strength of neuronal recurrent interactions is increased. This localized state consists of a single narrow peak of activity whose center can lock to a slowly rotating, weakly-tuned external stimulus. Interestingly, we find that the bifurcation from the resting state is subcritical, i.e.: (i) the system jumps to a localized activity profile on destabilization of the resting state and (ii) hysteresis occurs in the sense that sharp orientation tuning can coexist with a stable resting state.

We then turn to the full IF model and show how an analysis of the nonlinear firing time map can serve as a basis for understanding orientation tuning in networks of spiking neurons. We derive explicit criteria for the stability of phase-locked solutions by considering the propagation of perturbations of the firing times throughout the network. Our analysis identifies regions in parameter space where instabilities in the firing times cause (subcritical) bifurcations to nonphase-locked states that support localized patterns of mean firing rates across the network similar to the sharp orientation tuning curves found in the corresponding analog model. However, as found in the case of global pattern formation (Bressloff and Coombes 1998b, 1999), the tuning 
curves are modulated by deterministic fluctuations of the ISIs on closed quasiperiodic orbits, which grow with the speed of synapses. For sufficiently fast synapses, the resulting coefficient of variation $\left(C_{V}\right)$ can be of order unity and the ISIs appear to exhibit chaotic motion due to break-up of the quasiperiodic orbits.

\section{The model}

We consider an integrate-and-fire version of the neural network model for orientation tuning in a cortical hypercolumn developed by Hansel and Sompolinsky (1997) and Ben-Yishai et al (1997). This is a simplified version of the model studied numerically by Somers et al (1995). The network consists of two sub-populations of neurons, one excitatory and the other inhibitory, which code for the orientation of a visual stimulus appearing in a common visual field (see figure 1). The index $L=E, I$ will be used to distinguish between the two populations. Each neuron is parametrized by an angle $\phi, 0 \leq \phi<\pi$, which represents its orientation preference. (The angle is restricted to be from 0 to $\pi$ since a bar that is oriented at an angle 0 is indistinguishable from one that has an orientation $\pi)$. Let $U_{L}(\phi, t)$ denote the membrane potential at time $t$ of a neuron of type $L$ and orientation preference $\phi$. The neurons are modeled as IF oscillators evolving according to the set of equations

$$
\tau_{0} \frac{\partial U_{L}(\phi, t)}{\partial t}=h_{0}-U_{L}(\phi, t)+X_{L}(\phi, t), \quad L=E, I
$$

where $h_{0}$ is a constant external input or bias, $\tau_{0}$ is a membrane time constant, and $X_{L}(\phi, t)$ denotes the total synaptic input to a neuron $\phi$ of type $L$. Periodic boundary conditions are assumed so that $U_{L}(0, t)=U_{L}(\pi, t)$. Equation (2.1) is supplemented by the condition that whenever a neuron reaches a threshold $\kappa$ it fires a spike and its membrane potential is immediately reset to zero. In other words,

$$
U_{L}\left(\phi, t^{+}\right)=0 \quad \text { whenever } \quad U_{L}(\phi, t)=\kappa, \quad L=E, I
$$

For concreteness, we set the threshold $\kappa=1$ and take $h_{0}=0.9<\kappa$ so that in the absence of any synaptic inputs all neurons are quiescent. We also fix the fundamental unit of time to be of the order $10 \mathrm{msec}$ by setting $\tau_{0}=1$. (All results concerning firing rates or inter-spike intervals presented in this paper are in units of $\left.\tau_{0}\right)$. 


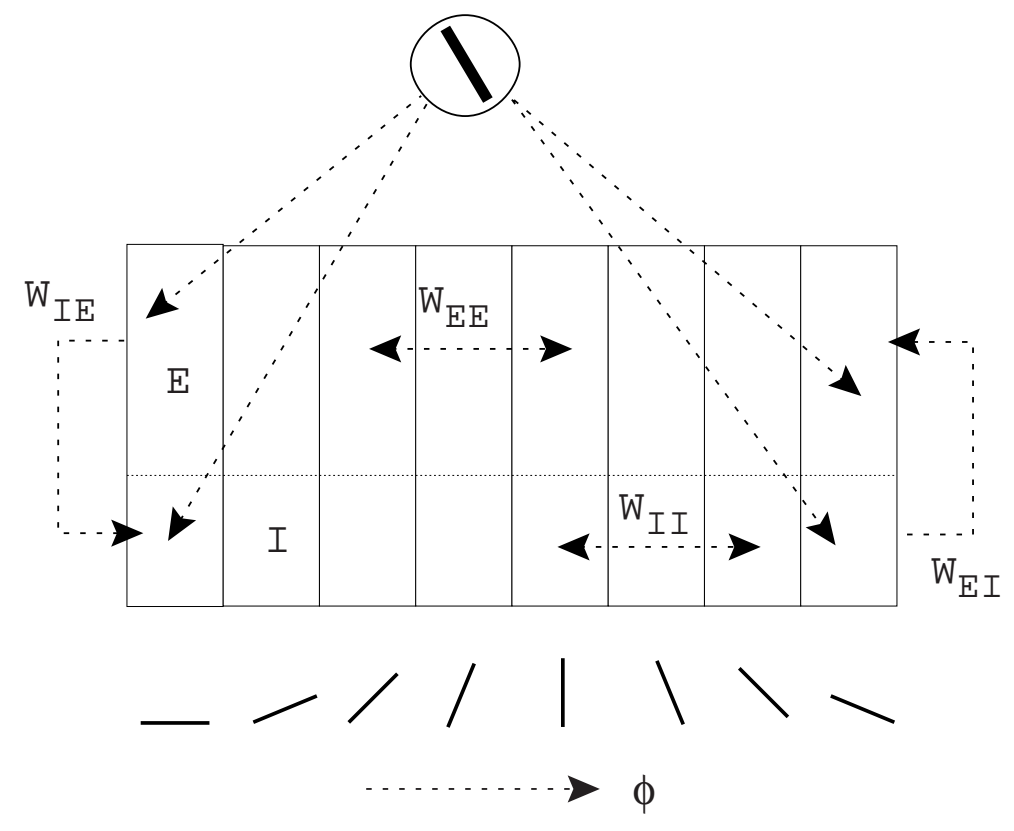

Figure 1: Network architecture of a ring model of a cortical hypercolumn (see Hansel and Sompolinsky (1997))

The total synaptic input $X_{L}(\phi, t)$ is taken to be of the form

$$
X_{L}(\phi, t)=\sum_{M=E, I} \int_{0}^{\pi} \frac{d \phi^{\prime}}{\pi} W_{L M}\left(\phi-\phi^{\prime}\right) Y_{M}\left(\phi^{\prime}, t\right)+h_{L}(\phi, t)
$$

where $W_{L M}\left(\phi-\phi^{\prime}\right)$ denotes the interaction between a presynaptic neuron $\phi^{\prime}$ of type $M$ and a postsynaptic neuron $\phi$ of type $L$, and $Y_{M}\left(\phi^{\prime}, t\right)$ is the effective input at time $t$ induced by the incoming spike train from the presynaptic neuron (also known as the synaptic drive, Pinto, Brumberg, Simons and Ermentrout 1996). The term $h_{L}(\phi, t)$ represents the inputs from the lateral geniculate nucleus (LGN). The weight functions $W_{L M}$ are $\pi$-periodic in $\phi$. The interactions are assumed to depend on the degree of similarity of the presynaptic and postsynaptic orientation preferences, and to be of maximum strength when they have the same preference. Following Hansel and Sompolinsky (1997), we choose the interactions to have the particular form

$$
\begin{array}{ll}
W_{L E}(\phi)=W_{0}^{L E}+2 W_{2}^{L E} \cos (2 \phi), & W_{0}^{L E} \geq 2 W_{2}^{L E} \geq 0 \\
W_{L I}(\phi)=-W_{0}^{L I}-2 W_{2}^{L I} \cos (2 \phi), & W_{0}^{L I} \geq 2 W_{2}^{L I} \geq 0
\end{array}
$$


Finally, we take

$$
Y_{L}(\phi, t)=\int_{0}^{\infty} d \tau \rho(\tau) f_{L}(\phi, t-\tau)
$$

where $\rho(\tau)$ represents some delay distribution and $f_{L}(\phi, t)$ is the output spike train of a neuron $\phi$ of type $L$. Neglecting the pulse-shape of an individual action potential, we represent the output spike train as a sequence of impulses,

$$
f_{L}(\phi, t)=\sum_{k \in \mathbb{Z}} \delta\left(t-T_{L}^{k}(\phi)\right)
$$

where $T_{L}^{k}(\phi)$, integer $k$, denotes the $k$ th firing time (threshold-crossing time) of the given neuron. The delay distribution $\rho(\tau)$ can incorporate a number of possible sources of delay in neural systems: (i) Discrete delays arising from finite axonal transmission times. (ii) Synaptic processing delays associated with the conversion of an incoming spike to a post-synaptic potential. (iii) Dendritic processing delays in which the effects of a post-synaptic potential generated at a synapse located on the dendritic tree at some distance from the soma is mediated by diffusion along the tree. For concreteness, we shall restrict ourselves to synaptic delays and take $\rho(t)$ to be an alpha-function (Destexhe, Mainen and Sejnowski 1994)

$$
\rho(\tau)=\alpha^{2} \tau \mathrm{e}^{-\alpha \tau} \Theta(\tau)
$$

where $\alpha$ is the inverse rise-time of a post-synaptic potential, and $\Theta(\tau)=1$ if $\tau \geq 0$ and is zero otherwise. We expect axonal delays to be small within a given hypercolumn. (For a review of the dynamical effects of dendritic structure, see Bressloff and Coombes 1997).

It is important to see how the above spiking version of the ring model is related to the rate models considered by Hansel and Sompolinsky (1997) and Ben-Yishai et al (1997). Suppose the synaptic interactions are sufficiently slow so that the output $f_{L}(\phi, t)$ of a neuron can be characterized reasonably well by a mean (time-averaged) firing rate (see for example Amit and Tsodyks 1991; Bressloff and Coombes 1999). Let us consider the case in which $\rho(\tau)$ is given by the alpha-function (2.8) with a synaptic rise time $\alpha^{-1}$ significantly longer than all other time-scales in the system. The total synaptic input to neuron $\phi$ of type $L$ will then be described by a slowly time-varying function $X_{L}(\phi, t)$ such that the actual firing rate will quickly relax to 
approximately the steady state value, as determined by equations (2.1) and (2.2). This implies that

$$
f_{L}(\phi, t)=f\left(X_{L}(\phi, t)\right)
$$

with the steady-state firing-rate function $f$ given by

$$
f(X)=\left\{\ln \left[\frac{h_{0}+X}{h_{0}+X-1}\right]\right\}^{-1} \Theta\left(h_{0}+X-1\right)
$$

(For the sake of simplicity, we shall ignore the effects of refractory period, which is reasonable when the system is operating well below its optimal firing rate). Equation (2.9) relates the dynamics of the firing rate directly to the stimulus dynamics $X_{L}(\phi, t)$ through the steady-state response function. In effect, the use of a slowly varying distribution $\rho(\tau)$ allows a consistent definition of the firing rate so that a dynamical network model can be based upon the steady state properties of an isolated neuron.

Substitution of equations (2.6) and (2.9) into (2.3) yields the extended Wilson-Cowan equations (Wilson and Cowan 1973)

$$
X_{L}(\phi, t)=\sum_{M=E, I} \int_{0}^{\pi} \frac{d \phi^{\prime}}{\pi} W_{L M}\left(\phi-\phi^{\prime}\right) \int_{0}^{\infty} d \tau \rho(\tau) f\left(X_{M}\left(\phi^{\prime}, t-\tau\right)\right)+h_{L}(\phi, t)
$$

An alternative version of equation (2.11) may be obtained for the alpha-function delay distribution (2.8), by rewriting equation (2.6) as the differential equation

$$
\frac{1}{\alpha^{2}} \frac{\partial^{2} Y_{L}}{\partial t^{2}}+\frac{2}{\alpha} \frac{\partial Y_{L}}{\partial t}+Y_{L}=f\left(X_{L}\right)
$$

with $X_{L}$ given by equation (2.3), and $Y_{L}(\phi, t), \partial Y_{L}(\phi, t) / \partial t \rightarrow 0$ as $t \rightarrow-\infty$. Similarly taking $\rho(t)=\alpha \mathrm{e}^{-\alpha t}$ generates the particular version of the Wilson-Cowan equations studied by Hansel and Sompolinsky (1997),

$$
\alpha^{-1} \frac{\partial Y_{L}}{\partial t}+Y_{L}=f\left(X_{L}\right)
$$

There are a number of differences, however, between the interpretation of $Y_{L}$ in equation (2.13) and the corresponding variable considered by Hansel and Sompolinsky (1997). In the former case, $Y_{L}(\phi, t)$ is the synaptic drive of a single neuron with orientation preference $\phi$ and $f$ is a time-averaged firing-rate, whereas in the latter case $Y_{L}$ represents the activity of a population of 
neurons forming an orientation column $\phi$ and $f$ is some gain function. It is possible to introduce the notion of an orientation column in our model by partitioning the domain $0 \leq \phi<\pi$ into $N$ segments of length $\pi / N$ such that

$$
m_{L}\left(\phi_{k}, t\right)=N \int_{k \pi / N}^{(k+1) \pi / N} \frac{d \phi}{\pi} f_{L}(\phi, t), \quad k=0,1, \ldots, N-1
$$

represents the population-averaged firing-rate within the $k$ th orientation column, $\phi_{k}=k \pi / N$. (Alternatively, we could reinterpret the IF model as a caricature of a synchronized column of spiking neurons).

Hansel and Sompolinsky (1997) and Ben-Yishai et al $(1995,1997)$ have carried out a detailed investigation of orientation tuning in the mean firing rate version of the ring model defined by equations (2.3) and (2.13) with $f$ a semilinear gain function. They consider external inputs of the form

$$
h_{L}(\phi, t)=C \Gamma_{L}\left[1-\epsilon+\epsilon \cos \left(\phi-\phi_{0}(t)\right)\right]
$$

for $0 \leq \epsilon \leq 0.5$. This represents a tuned input from the LGN due to a visual stimulus of orientation $\phi_{0}$. The parameter $C$ denotes the contrast of the stimulus, $\Gamma_{L}$ is the transfer function from the LGN to the cortex, and $\epsilon$ determines the angular anisotropy. In the case of a semilinear gain function, $f(x)=0$ if $x \leq 0, f(x)=x$ if $x \geq 0$ and $f(x)=1$ for $x \geq 1$, it is possible to derive self-consistency equations for the activity profile of the network. Solution of these equations shows that in certain parameter regimes local cortical feedback can generate sharp orientation tuning curves in which only a fraction of neurons are active. The associated activity profile consists of a single peak centered about $\phi_{0}$ (Hansel and Sompolinsky 1997). This activity peak can also lock to a rotating stimulus $\phi_{0}=\Omega t$ provided that $\Omega$ is not too large; if the inhibitory feedback is sufficiently strong then it is possible for spontaneous wave propagation to occur even in the absence of a rotating stimulus (Ben-Yishai et al 1997).

The idea that local cortical interactions play a central role in generating sharp orientation tuning curves is still controversial. The classical model of Hubel and Wiesel (1962) proposes a very different mechanism, in which the orientation preference of a cell arises primarily from the geometrical alignment of the receptive fields of the LGN neurons projecting to it. A number of recent experiments show a significant correlation between the alignment of receptive fields 
of LGN neurons and the orientation preference of simple cells functionally connected to them (Chapman, Zahs and Stryker 1991; Reid and Alonso 1996). In addition, Ferster, Chung and Wheat (1996) have shown that cooling a patch of cortex and therefore presumably abolishing cortical feedback does not totally abolish the orientation tuning exhibited by EPSPs generated by LGN input. However, there is also growing experimental evidence suggesting the importance of cortical feedback. For example, the blockage of extracellular inhibition in cortex leads to considerably less sharp orientation tuning (Sillito, Kemp, Milson and Beradi 1980; Ferster and Koch 1987; Nelson, Toth, Seth and Mur 1994). Moreover, intracellular measurements indicate that direct inputs from the LGN to neurons in layer 4 of the primary visual cortex provide only a fraction of the total excitatory inputs relevant to orientation selectivity (Pei, Vidyasagar, Volgushev and Creutzseldt 1994; Douglas, Koch, Mahowald, Martin and Suarez 1995). See also the discussion in Somers et al (1995). In addition, there is evidence that orientation tuning takes about 50-60 msec. to reach its peak, and that the dynamics of tuning has a rather complex time course (Ringach, Hawken and Shapley 1997) suggesting some cortical involvement.

Recently Ermentrout (1998) has pointed out that the dynamical mechanism for sharp orientation tuning identified by Hansel and Sompolinsky (1997) can be interpreted as a localized form of spontaneous pattern formation (at least for strongly modulated cortical interactions). For general spatially distributed systems, pattern formation concerns the loss of stability of a spatially uniform state through a bifurcation to a spatially periodic state (Murray 1990). The latter may be stationary or oscillatory (time-periodic). Pattern formation in neural networks was first studied in detail by Ermentrout and Cowan (1979a,b). They showed how competition between short-range excitation and long-range inhibition in a two-dimensional Wilson-Cowan network can induce periodic striped and hexagonal patterns of activity. These spontaneous patterns provided a possible explanation for the generation of visual hallucinations. See also Cowan (1982). At first sight, the analysis of orientation tuning by Hansel and Sompolinsky (1997) and Ben-Yishai et al (1997) appears to involve a different dynamical mechanism from this, since only a single peak of activity is formed over the domain $0 \leq \phi \leq \pi$ rather than a spatially repeating pattern. This apparent difference vanishes, however, once it is realized that spatially periodic patterns would be generated by "unravelling" the ring. Thus, the one-dimensional stationary 
and propagating activity profiles found by Wilson and Cowan (1973) correspond respectively to stationary and time-periodic patterns on the $\pi$-periodic ring. In the following sections we study orientation tuning in both the analog and IF models from the viewpoint of spontaneous pattern formation.

\section{Orientation tuning in analog model}

We first consider orientation tuning in the analog or rate model described by equation (2.11) with the firing rate function given by (2.10). This may be viewed as the $\alpha \rightarrow 0$ limit of the IF model. We shall initially restrict ourselves to the case of time-independent external inputs $h_{L}(\phi)$. Given a $\phi$-independent input $h_{L}(\phi)=C_{L}$, the homogeneous equation (2.11) has at least one spatially uniform solution $X_{L}(\phi, t)=\bar{X}_{L}$ where

$$
\bar{X}_{L}=\sum_{M=E, I} W_{0}^{L M} f\left(\bar{X}_{M}\right)+C_{L}
$$

The local stability of the homogeneous state is found by linearizing equation (2.11) about $\bar{X}_{L}$. Setting $x_{L}(\phi, t)=X_{L}(\phi, t)-\bar{X}_{L}$ and expanding to first-order in $x_{L}$ gives

$$
x_{L}(\phi, t)=\sum_{M=E, I} \gamma_{M} \int_{0}^{\pi} \frac{d \phi^{\prime}}{\pi} W_{L M}\left(\phi-\phi^{\prime}\right) \int_{0}^{\infty} d \tau \rho(\tau) x_{M}\left(\phi^{\prime}, t-\tau\right)
$$

where $\gamma_{M}=f^{\prime}\left(\bar{X}_{M}\right)$ and $f^{\prime} \equiv d f / d X$. Equation (3.2) has solutions of the form

$$
x_{L}(\phi, t)=Z_{L} \mathrm{e}^{ \pm \nu t} \mathrm{e}^{i n \phi}, \quad n=0,2
$$

For each $n$, the eigenvalues $\nu$ and corresponding eigenvectors $\mathbf{Z}=\left(Z_{E}, Z_{I}\right)^{\text {tr }}$ satisfy the equation

$$
\mathbf{Z}=\widetilde{\rho}(\nu) \widehat{\mathbf{W}}_{n} \mathbf{Z}
$$

where

$$
\widetilde{\rho}(\nu) \equiv \int_{0}^{\infty} \mathrm{e}^{-\nu \tau} \rho(\tau) d \tau=\frac{\alpha^{2}}{(\alpha+\nu)^{2}}
$$

for the alpha-function (2.8), and

$$
\widehat{\mathbf{W}}_{n}=\left(\begin{array}{cc}
\gamma_{E} W_{n}^{E E} & -\gamma_{I} W_{n}^{E I} \\
\gamma_{E} W_{n}^{I E} & -\gamma_{I} W_{n}^{I I}
\end{array}\right)
$$


The state $\bar{X}_{L}$ will be stable if all eigenvalues $\nu$ have a negative real part. It follows that the stability of the homogeneous state depends on the particular choice of weight parameters $W_{0}^{L M}, W_{2}^{L M}$ and the external inputs $C_{L}$, which determine the factors $\gamma_{L}$. On the other hand, stability is independent of the inverse rise-time $\alpha$. (This $\alpha$-independence will no longer hold for the IF model, see section 4).

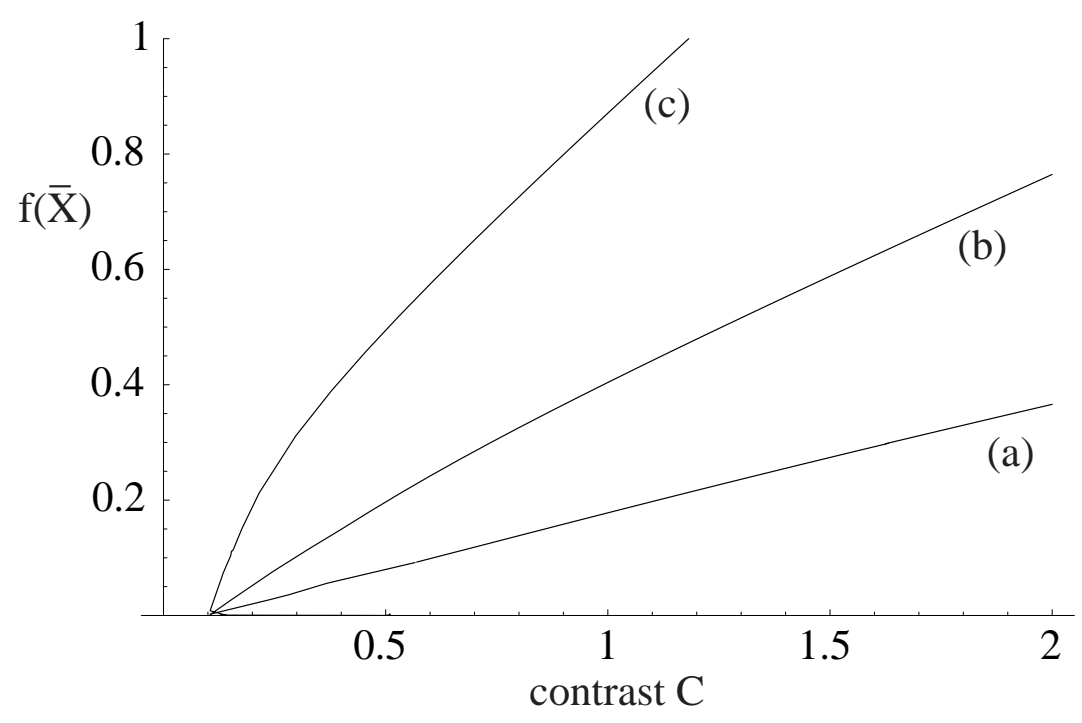

Figure 2: Plot of steady-state firing-rate $f(\bar{X})$ as a function of the contrast $C$ for various choices of weight parameters: (a) $W_{0}=-5$, (b) $W_{0}=-2$, (c) $W_{0}=-0.5$ where $W_{0}=W_{0}^{E}-W_{0}^{I}$.

In order to simplify matters further, let us consider a symmetric two-population model in which

$$
C_{L}=C, \quad W_{n}^{E E}=W_{n}^{I E} \equiv W_{n}^{E}, \quad W_{n}^{E I}=W_{n}^{I I} \equiv W_{n}^{I}
$$

Equation (3.1) then has a solution $X_{L}=\bar{X}$ where $\bar{X}$ is the unique solution of the equation $\bar{X}=W_{0} f(\bar{X})+C$ (see figure 2). We shall assume that $C$ is superthreshold, $h_{0}+C>1$, otherwise $\bar{X}=C$ such that $f(\bar{X})=0$. Moreover, $\gamma_{L}=\gamma \equiv f^{\prime}(\bar{X})$ for $L=E$, $I$ so that $\widehat{\mathbf{W}}_{n}=\gamma \mathbf{W}_{n}$. Equations (3.4)-(3.6) then reduce to the eigenvalue equation

$$
\left(\frac{\nu}{\alpha}+1\right)^{2}=\gamma \lambda_{n}^{ \pm}
$$

for $n=0,2$, where $\lambda_{n}^{ \pm}$are the eigenvalues of the weight matrix $\mathbf{W}_{n}$ with corresponding eigen- 
vectors $\mathbf{Z}_{n}^{ \pm}$:

$$
\lambda_{n}^{+}=W_{n} \equiv W_{n}^{E}-W_{n}^{I}, \quad \lambda_{n}^{-}=0, \quad \mathbf{Z}_{n}^{+}=\left(\begin{array}{c}
1 \\
1
\end{array}\right), \quad \mathbf{Z}_{n}^{-}=\left(\begin{array}{c}
1 / W_{n}^{E} \\
1 / W_{n}^{I}
\end{array}\right)
$$

It follows from equation (3.8) that $\bar{X}$ is stable with respect to excitation of the $(-)$ modes $\mathbf{Z}_{0}^{-}, \mathbf{Z}_{2}^{-} \cos (2 \phi), \mathbf{Z}_{2}^{-} \sin (2 \phi)$. If $\gamma W_{0}<1$ and $\gamma W_{2}<1$ then $\bar{X}$ is also stable with respect to excitation of the corresponding $(+)$ modes. This implies that the effect of the superthreshold LGN input $h_{L}(\phi)=C$ is to switch the network from an inactive state to a stable homogeneous active state. In this parameter regime, sharp orientation tuning curves can only be generated if there is a sufficient degree of angular anisotropy in the afferent inputs to the cortex from the LGN, that is, $\epsilon$ in equation (2.15) must be sufficiently large (Hansel and Sompolinsky 1997). This is the classical model of orientation tuning (Hubel and Wiesel 1962).

An alternative mechanism for sharp orientation tuning can be obtained by having strongly modulated cortical interactions such that $\gamma W_{0}<1$ and $\gamma W_{2}>1$. The homogeneous state then destabilizes due to excitation of the first harmonic modes $\mathbf{Z}_{2}^{+} \cos (2 \phi), \mathbf{Z}_{2}^{+} \sin (2 \phi)$. Since these modes have a single maximum in the interval $(0, \pi)$, we expect the network to support an activity profile consisting of a solitary peak centered about some angle $\phi_{0}$, which is the same for both the excitatory and inhibitory populations. For $\phi$-independent external inputs $(\epsilon=0)$, the location of this center is arbitrary, which reflects the underlying translational invariance of the network. Following Hansel and Sompolinsky (1997), we call such an activity profile a marginal state. The presence of a small angular anisotropy in the inputs $(0<\epsilon \ll 1$ in equation (2.15)) breaks the translational invariance of the system and locks the location of the center to the orientation corresponding to the peak of the stimulus (Hansel and Sompolinsky 1997, Ben-Yishai et al 1997). In contrast to afferent mechanisms of sharp orientation tuning, $\epsilon$ can be arbitrarily small.

The critical coupling at which the marginal state becomes excited is determined from the condition $W_{2}=W_{2 c} \equiv 1 / f^{\prime}(\bar{X})$. This generates a phase boundary curve in the $\left(W_{0}, W_{2}\right)$-plane that separates a stable homogeneous state and a marginal state. (See solid curve in figure 3). A direct numerical study of the analog network shows that when the phase boundary is crossed from below, the network jumps to a stable marginal state consisting of a sharp orientation tuning 
curve. The width of the activity profile is parameter-independent, whereas the height increases with $W_{2}$ for example (see figure 4). Note, however, that the marginal state is itself destroyed by amplitude instabilities if $W_{2}$ becomes too large. Consistent with the results of Hansel and Sompolinsky (1997) and Ben-Yishai et al (1997), the center of the activity peak can lock to a slowly rotating weakly-tuned stimulus as illustrated in figure 5 .

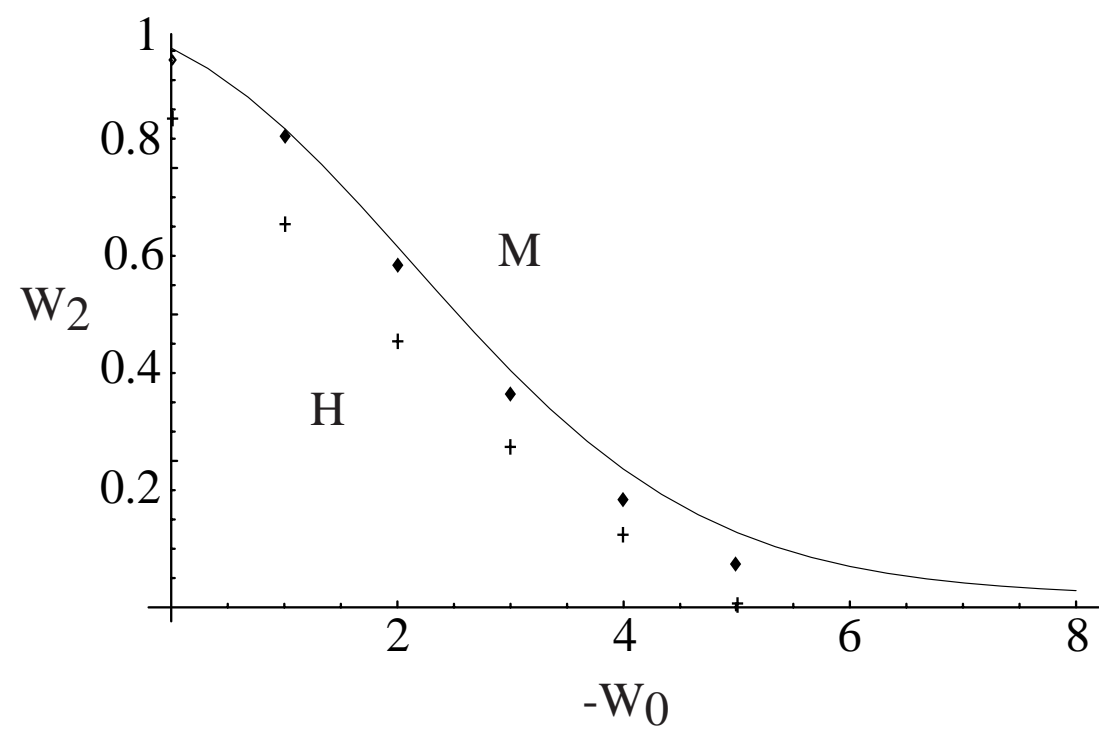

Figure 3: Phase boundary in the $\left(W_{0}, W_{2}\right)$-plane (solid curve) between a stable homogeneous state $(H)$ and a stable marginal state $(M)$ of the analog model for $C=1$. Also shown are data points from a direct numerical simulation of a symmetric two-population network consisting of $N=100$ neurons per population: (a) critical coupling for destabilization of homogeneous state (solid diamonds) and (b) lower critical coupling for persistence of stable marginal state due to hysteresis (crosses).

Interestingly, the marginal state is found to exhibit hysteresis in the sense that sharp orientation tuning persists for a range of values of $W_{2}<W_{2 c}$. That is, over a certain parameter regime a stable homogeneous state and a stable marginal state coexist (see data points in figure 3). This suggests that the bifurcation is subcritical, which is indeed found to be the case as we now explain. In standard treatments of pattern formation, bifurcation theory is used to derive nonlinear ODEs for the amplitude of the excited modes from which existence and stability can be determined, at least close to the bifurcation point (Cross and Hohenberg 1993, Ermen- 


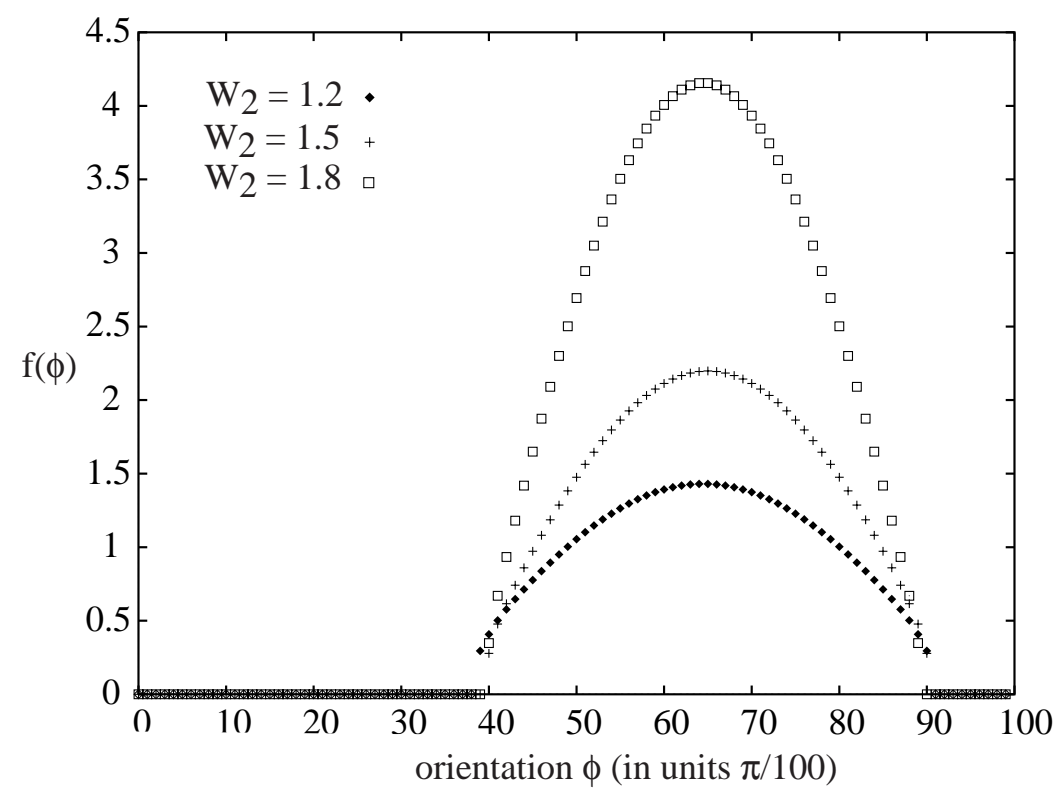

Figure 4: Sharp orientation tuning curves for a symmetric two-population analog network $(N=$ 100). The firing rate $f(\phi)$ is plotted as a function of orientation for $W_{2}=1.2,1.5,1.8$. Other parameter values are $W_{0}=-0.1, C=0.25$, and $\alpha=0.5$.
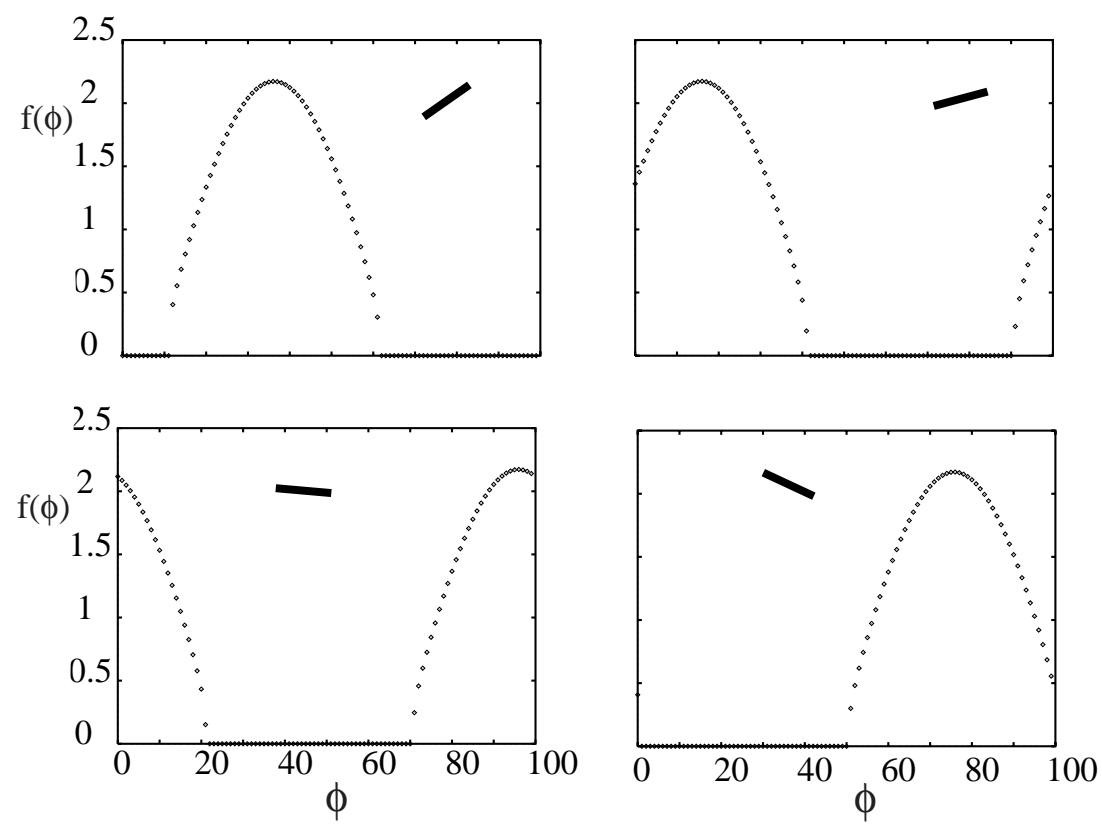

Figure 5: Orientation tuning curve in a symmetric two-population analog network $(N=100)$ locking to a slowly rotating, weakly-tuned external stimulus. Here $W_{0}=-0.1, W_{2}=1.5$, $C=0.25, \alpha=1.0$. The degree of angular anisotropy in the input is $\epsilon=0.1$ and the angular frequency of rotation is $\Omega=0.0017$ (which corresponds to approximately $10^{\circ}$ per second) 
trout 1998). From the symmetries imposed by equations (3.7), we can restrict ourselves to the class of solutions $X_{L}(t)=X(t), L=E, I$, such that equation (2.11) reduces to the effective one-population model

$$
X(\phi, t)=\int_{0}^{\pi} \frac{d \phi^{\prime}}{\pi} W\left(\phi-\phi^{\prime}\right) \int_{0}^{\infty} d \tau \rho(\tau) f\left(X\left(\phi^{\prime}, t-\tau\right)\right)+C
$$

with $W(\phi)=W_{0}+2 W_{2} \cos (2 \phi)$. Suppose that the firing-rate function $f$ is expanded about the (non-zero) fixed point $\bar{X}$,

$$
f(X)-f(\bar{X})=\gamma(X-\bar{X})+g_{2}(X-\bar{X})^{2}+g_{3}(X-\bar{X})^{3}+\ldots
$$

where $\gamma=f^{\prime}(\bar{X}), g_{2}=f^{\prime \prime}(\bar{X}) / 2, g_{3}=f^{\prime \prime \prime}(\bar{X}) / 6$. Let $W_{2}$ be a bifurcation parameter (for fixed $\left.W_{0}, C\right)$ with $W_{2}-W_{2 c}=\epsilon^{2}$, and $W_{2 c}=1 / \gamma$ the critical value for excitation of a marginal state. Substitute into equation (3.10) the perturbation expansion

$$
X(\phi, t)-\bar{X}=\left[Z(t) \mathrm{e}^{2 i n \phi}+Z^{*}(t) \mathrm{e}^{-2 i n \phi}\right]+\mathcal{O}\left(\epsilon^{2}\right)
$$

where $Z(t), Z^{*}(t)$ denote a complex conjugate pair of amplitudes.

A standard perturbation calculation (see appendix A and Ermentrout 1998) shows that $Z(t)$ evolves according to an equation of the form

$$
\frac{d Z}{d t}=Z\left(\gamma^{2}\left[W_{2}-W_{2 c}\right]+A|Z|^{2}\right)
$$

where

$$
A=3 g_{3}+\frac{4 g_{2}^{2} W_{0}}{1-\gamma W_{0}}
$$

Since $W_{2}, \gamma$ and $A$ are all real, the phase of $Z$ is arbitrary (reflecting a marginal state) whereas the amplitude is given by $|Z|=\sqrt{\gamma^{2}\left|W_{2}-W_{2 c}\right| / A}$. It is clear that a stable marginal state will bifurcate from the homogeneous state if and only if $A<0$. A quick numerical calculation shows that $A>0$ for all $W_{0}$ and $C$. This implies that the bifurcation is subcritical, that is, the resting state bifurcates to an unstable broadly tuned orientation curve whose amplitude is $\mathcal{O}(\epsilon)$. Since the bifurcating tuning curve is unstable it is not observed in practice, rather one finds that the system jumps to a stable, large amplitude, sharp tuning curve which coexists with the resting state, and leads to hysteresis effects as illustrated schematically in figure 6 . 
Note that in the case of sigmoidal firing rate functions $f(X)$, the bifurcation may be either sub- or super-critical, depending on the relative strengths of recurrent excitation and lateral inhibition (Ermentrout and Cowan 1979b). If the latter is sufficiently strong, then supercritical bifurcations can occur in which the resting state changes smoothly into a sharply tuned state. However in the case studied here the nonlinear firing rate function $f(X)$ of equation $(2.10)$ is such that the bifurcation is almost always subcritical.

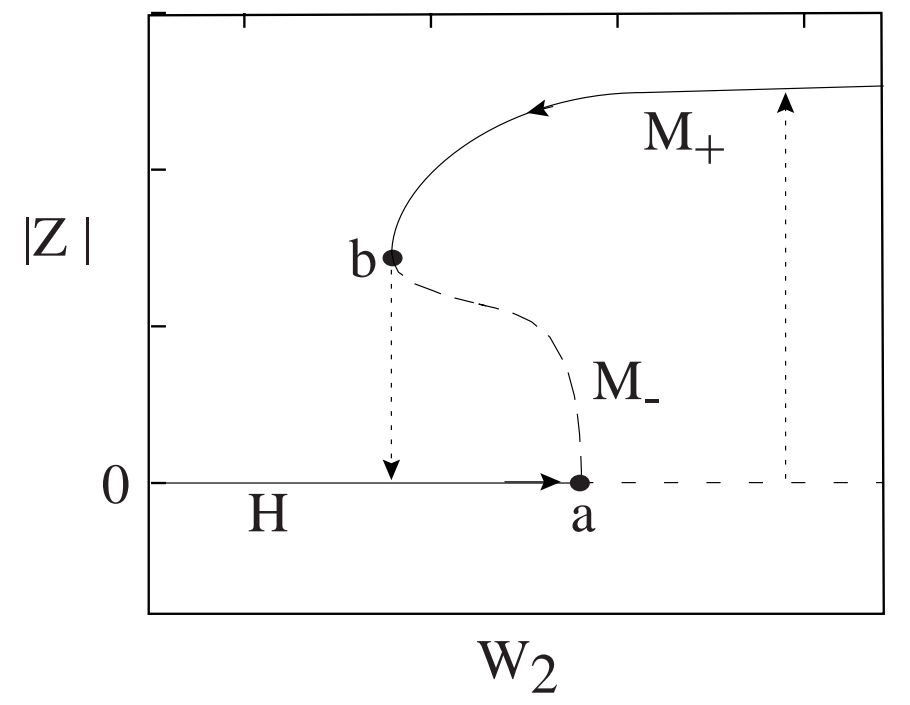

Figure 6: Schematic diagram illustrating hysteresis effects in the analog model. As the coupling parameter $W_{2}$ is slowly increased from zero a critical point $a$ is reached where the homogeneous resting state $(H)$ becomes unstable and there is a subcritical bifurcation to an unstable, broadly tuned marginal state $\left(M_{-}\right)$. Beyond the bifurcation point, the system jumps to a stable, sharply tuned marginal state $\left(M_{+}\right)$which coexists with the other solutions. If the coupling $W_{2}$ is now slowly reduced the marginal state $M_{+}$persists beyond the original point $a$ so that there is a range of values $W_{2}(b)<W_{2}<W_{2}(a)$ over which a stable resting state co-exists with a stable marginal state (bistability). At point $b$ the states $M_{ \pm}$annihilate each other and the system jumps back to the resting state. 


\section{Orientation tuning in IF model}

Our analysis of the analog model in section 3 ignored any details concerning neural spike trains by taking the output of a neuron to be an average firing-rate. We now return to the more realistic IF model of equations (2.1)-(2.3) in which the firing-times of individual spikes is specified. We wish to identify the analogous mechanism for orientation tuning in the model with spike-coding.

\subsection{Existence and stability of synchronous state}

The first step is to specify what is meant by a homogeneous activity state. We define a phaselocked solution to be one in which every oscillator resets or fires with the same collective period $T$ that must be determined self-consistently. The state of each oscillator can then be characterized by a constant phase $\eta_{L}(\phi)$ with $0 \leq \eta_{L}(\phi)<1$ and $0 \leq \phi<\pi$. The corresponding firing times satisfy $T_{L}^{k}(\phi)=\left(k-\eta_{L}(\phi)\right) T$, integer $k$. Integrating equation (2.1) between two successive firing events and incorporating the reset condition (2.2) leads to the phase equations

$$
1=\left(1-\mathrm{e}^{-T}\right)\left(h_{0}+C_{L}\right)+\sum_{M=E, I} \int_{0}^{\pi} \frac{d \phi^{\prime}}{\pi} W_{L M}\left(\phi-\phi^{\prime}\right) K_{T}\left(\eta_{M}\left(\phi^{\prime}\right)-\eta_{L}(\phi)\right), \quad L=E, I
$$

where

$$
K_{T}(\eta)=\mathrm{e}^{-T} \int_{0}^{T} d t \mathrm{e}^{t} \sum_{k \in \mathbb{Z}} \rho(t+(k+\eta) T)
$$

Note that for any phase-locked solution of equation (4.1), the distribution of network output activity across the network, as specified by the inter-spike intervals (ISIs)

$$
\Delta_{L}^{k}(\phi)=T_{L}^{k+1}(\phi)-T_{L}^{k}(\phi)
$$

is homogeneous since $\Delta_{L}^{k}(\phi)=T$ for all $k \in \mathbb{Z}, 0 \leq \phi<\pi, L=(E, I)$. In other words, each phase-locked state plays an analogous role to the homogeneous state $\bar{X}_{L}$ of the mean firing rate model. To simplify our analysis, we shall concentrate on instabilities of the synchronous state $\eta_{L}(\phi)=\bar{\eta}$ where $\bar{\eta}$ is an arbitrary constant. In order to ensure such a solution exists, we impose the conditions (3.7) so that (4.1) reduces to a single self-consistency equation for the collective period $T$ of the synchronous solution:

$$
1=\left(1-\mathrm{e}^{-T}\right) C_{0}+W_{0} K_{T}(0)
$$


where we have set $C_{0}=h_{0}+C$ and $W_{0}=W_{0}^{E}-W_{0}^{I}$. Solutions of equation (4.4) are plotted in figure 7 .

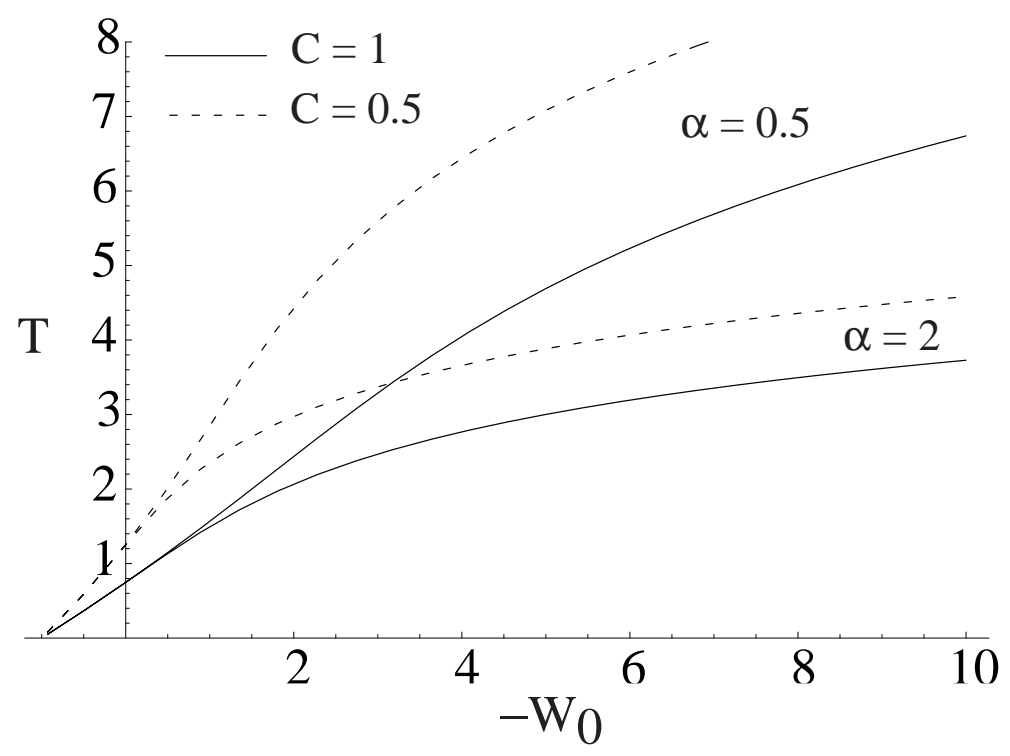

Figure 7: Variation of collective period $T$ of synchronous state as a function of $W_{0}$ for different contrasts $C$ and inverse rise-times $\alpha$.

The linear stability of the synchronous state can be determined by considering perturbations of the firing times (van Vreeswijk 1996; Gerstner, van Hemmen and Cowan 1996; Bressloff and Coombes 1998a,b, 1999)

$$
T_{L}^{k}(\phi)=(k-\bar{\eta}) T+\delta_{L}^{k}(\phi)
$$

Integrating equation (2.1) between two successive firing events yields a nonlinear mapping for the firing times which can be expanded as a power series in the perturbations $\delta_{L}^{k}(\phi)$. This generates a linear difference equation of the form

$$
A_{T}\left[\delta_{L}^{k+1}(\phi)-\delta_{L}^{k}(\phi)\right]=\sum_{l=0}^{\infty} G_{T}(l) \int_{0}^{\pi} \frac{d \phi^{\prime}}{\pi} \sum_{M=E, I} W_{L M}\left(\phi-\phi^{\prime}\right)\left[\delta_{M}^{k-l}\left(\phi^{\prime}\right)-\delta_{L}^{k}(\phi)\right]
$$

with

$$
G_{T}(l)=\mathrm{e}^{-T} \int_{0}^{T} d t \mathrm{e}^{t} \rho^{\prime}(t+l T)
$$




$$
A_{T}=C_{0}-1+W_{0} \sum_{k \in \mathbb{Z}} \rho(k T)
$$

Equation (4.6) has solutions of the form

$$
\delta_{L}^{k}(\phi)=\mathrm{e}^{k \nu} \mathrm{e}^{ \pm i n \phi} \delta_{L}, \quad n=0,2, \quad L=E, I
$$

where $\nu \in \mathbb{C}$ and $0 \leq \operatorname{Im} \nu<2 \pi$. The eigenvalues $\nu$ and associated eigenvectors $\mathbf{Z}=\left(\delta_{E}, \delta_{I}\right)^{\operatorname{tr}}$ for each $n=0,2$ satisfy the equation

$$
A_{T}\left[\mathrm{e}^{\nu}-1\right] \mathbf{Z}=\left[\widetilde{G}_{T}(\nu) \mathbf{W}_{n}-\widetilde{G}_{T}(0) \mathbf{D}\right] \mathbf{Z}
$$

where

$$
\widetilde{G}_{T}(\nu)=\sum_{k=0}^{\infty} G_{T}(k) \mathrm{e}^{-k \nu}
$$

and

$$
\mathbf{W}_{n}=\left(\begin{array}{cc}
W_{n}^{E E} & -W_{n}^{E I} \\
W_{n}^{I E} & -W_{n}^{I I}
\end{array}\right), \quad \mathbf{D}=\left(\begin{array}{cc}
W_{0}^{E E}-W_{0}^{E I} & 0 \\
0 & W_{0}^{I E}-W_{0}^{I I}
\end{array}\right)
$$

In order to simplify the subsequent analysis, we impose the same symmetry conditions (3.7) as used in the study of the analog model in section 3. We can then diagonalize (4.10) to obtain the result

$$
A_{T}\left[\mathrm{e}^{\nu}-1\right]=\widetilde{G}_{T}(\nu) \lambda_{n}^{ \pm}-\widetilde{G}_{T}(0) W_{0}
$$

where $\lambda_{n}^{ \pm}$are the eigenvalues of the weight matrix $\mathbf{W}_{n}$, see equation (3.9). Note that one solution of equation (4.13) is $\nu=0$ and $n=0$ associated with excitation of the uniform $(+)$ mode. This reflects invariance of the system with respect to uniform phase-shifts in the firing times. The synchronous state will be stable if all other solutions of equation (4.13) satisfy Re $\nu<0$. Following Bressloff and Coombes (1999), we investigate stability by first looking at the weak coupling regime in which $W_{n}=\mathcal{O}(\epsilon), n=0,2$, for some $\epsilon \ll 1$. Performing a perturbation expansion in $\epsilon$, it can be established that the stability of the synchronous state is determined by the non-zero eigenvalues in a small neighborhood of the origin, and these satisfy the equation (to first order in $\epsilon$ ) $A_{T} \nu=\left(\lambda_{n}^{ \pm}-W_{0}\right) \widetilde{G}_{T}(0)$. For the alpha-function (2.8), it can be established that $A_{T}>0$ whereas $\widetilde{G}_{T}(0) \equiv K_{T}^{\prime}(0) / T<0$. Therefore, the synchronous state 
is stable in the weak coupling regime provided that $W_{2}>W_{0}$ and $W_{0}<0$. Assuming that these two conditions are satisfied, we now investigate what happens as the strength of coupling is increased. First, it is easy to establish that the synchronous state cannot destabilize due to a real eigenvalue $\nu$ crossing the origin. In particular, it is stable with respect to excitations of the (-) modes. Thus any instability will involve one or more complex conjugate pairs of eigenvalues $\nu= \pm i \omega$ crossing the imaginary axis, signalling (for $\omega \neq \pi$ ) the onset of a discrete Hopf bifurcation (or Neimark-Sacker bifurcation) of the firing-times due to excitation of a $(+)$ mode. In the special case $\omega=\pi$ this reduces to a subharmonic or period doubling bifurcation since $\mathrm{e}^{ \pm i \pi}=-1$.

\subsection{Desynchronization leading to sharp orientation tuning}

In order to investigate the occurrence of a Hopf (or period-doubling) bifurcation in the firing times, substitute $\nu=i \omega$ into equation (4.13) for $\lambda_{n}^{+}=W_{n}$ and equate real and imaginary parts to obtain the pair of equations

$$
A_{T}[\cos (\omega)-1]=W_{n} \widetilde{C}(\omega)-W_{0} \widetilde{C}(0), \quad A_{T} \sin (\omega)=-W_{n} \widetilde{S}(\omega)
$$

where $n=0,2$ and

$$
\widetilde{C}(\omega)=\operatorname{Re} \widetilde{G}_{T}(i \omega), \widetilde{S}(\omega)=-\operatorname{Im} \widetilde{G}_{T}(i \omega)
$$

Explicit expressions for $\widetilde{C}(\omega)$ and $\widetilde{S}(\omega)$ in the case of the alpha function delay distribution (2.8) are given in appendix B. Suppose that $\alpha, W_{0}$ and $C_{0}$ are fixed with $T=T\left(\alpha, W_{0}, C_{0}\right)$ the unique self-consistent solution of equation (4.4), see figure 7. The smallest value of the coupling parameter, $W_{2}=W_{2 c}$, for which a non-zero solution, $\omega=\omega_{0} \neq 0$, of the simultaneous equations (4.15) is then sought. It turns out that such a solution occurs for $n=2$, signalling excitation of the eigenmodes $\delta_{L}^{k}(\phi)=\mathrm{e}^{ \pm i \omega_{0} k} \mathrm{e}^{ \pm i 2 \phi}, L=E, I$. This generates a phase boundary curve in the $\left(W_{0}, W_{2}\right)$-plane as illustrated in figures 8 and 9 for various $C$ and $\alpha$. Phase boundaries in the $\left(\alpha, W_{2}\right)$-plane and the $\left(C, W_{2}\right)$-plane are shown in figures 10 and 11 respectively. It should be noted that the cusps of the boundary curves in these figures simply correspond to points where two separate solution branches of equation (4.14) cross - only the lower branch is shown since this determines the critical coupling for destabilization of the synchronous state. The 


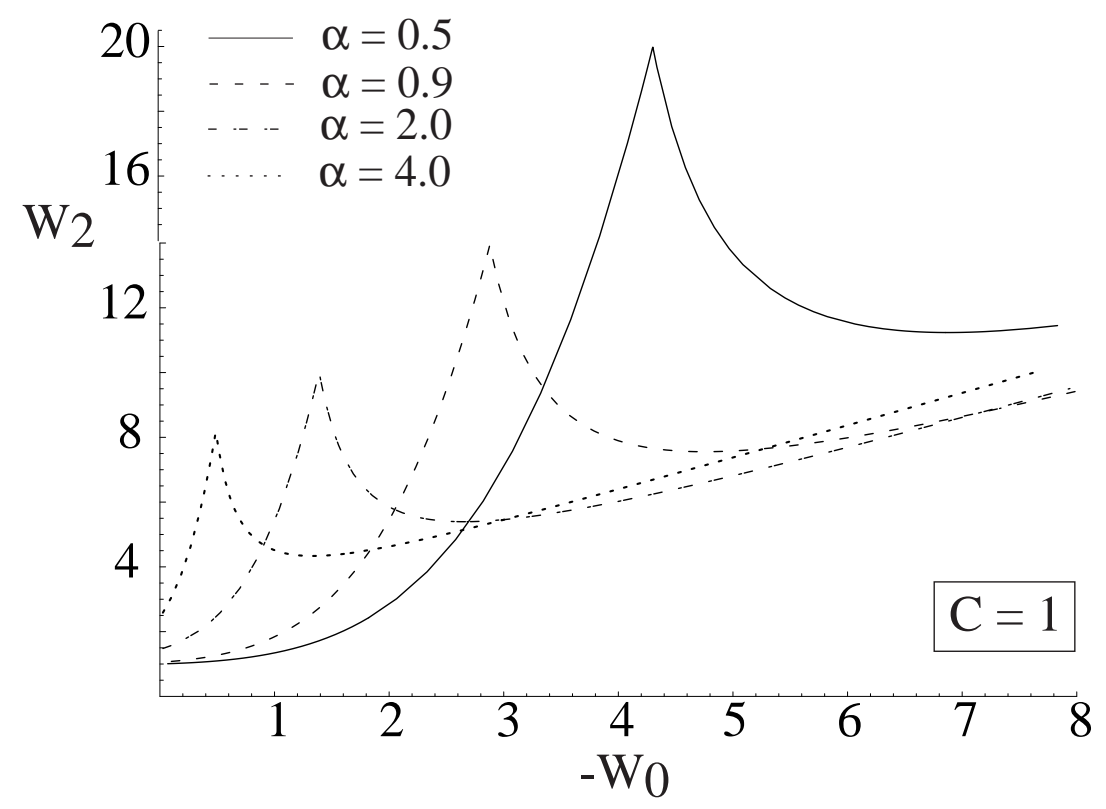

Figure 8: Phase boundary in the $\left(W_{0}, W_{2}\right)$-plane between a stable synchronous state and a marginal state of a symmetric two-population IF network. Boundary curves are shown for $C=1$ and various values of the inverse rise-time $\alpha$. In each case the synchronous sttae is stable below a given boundary curve.

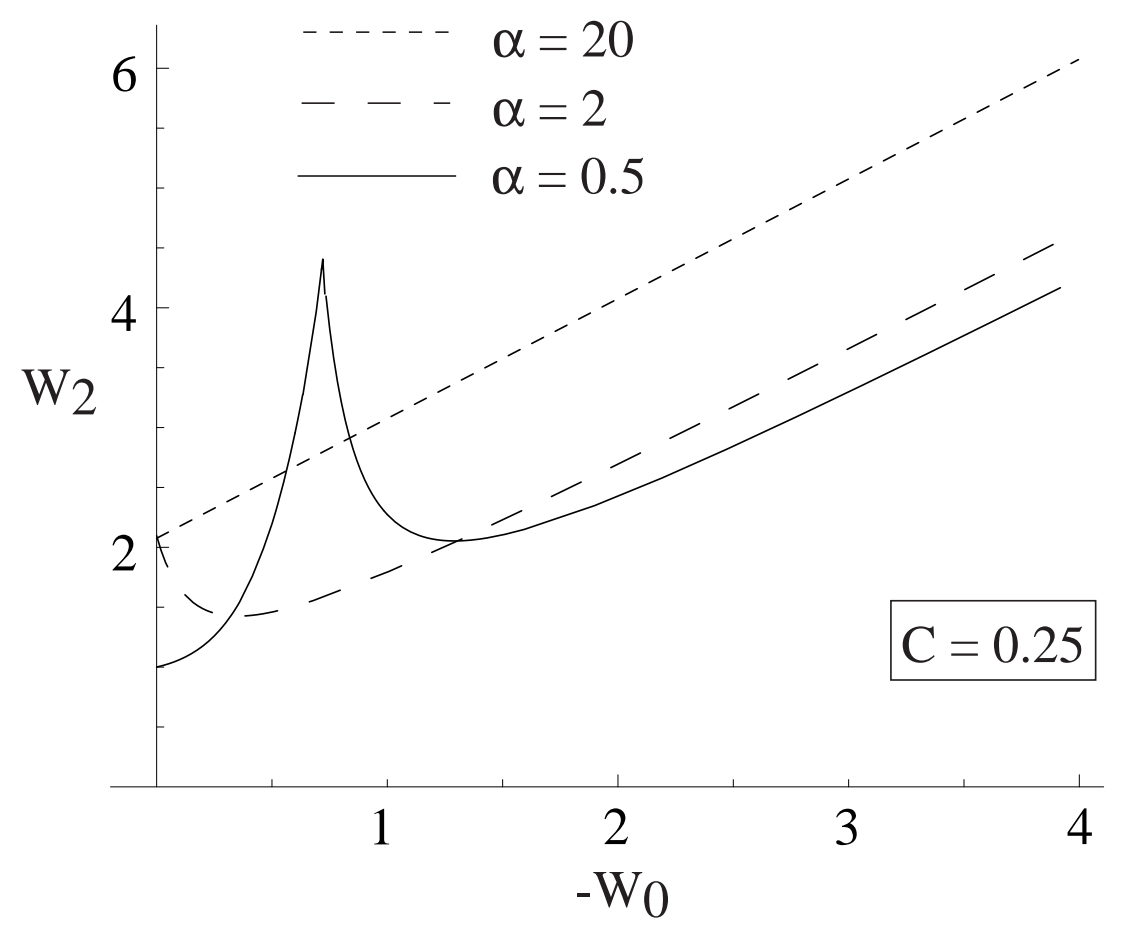

Figure 9: Same as figure 8 except that $C=0.25$. 


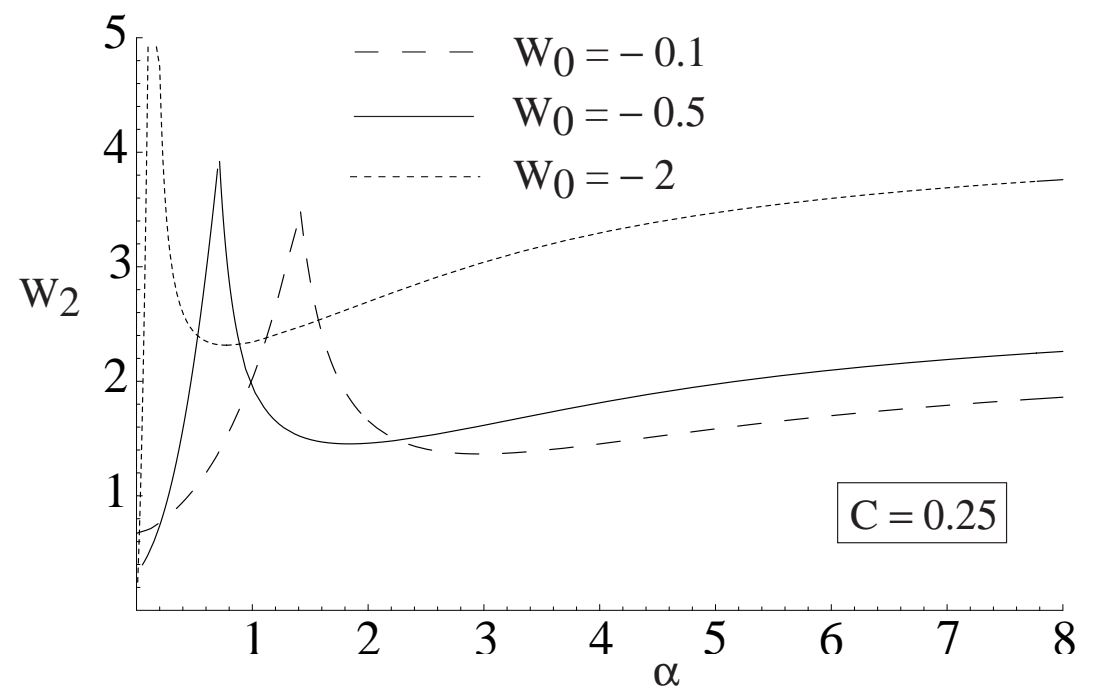

Figure 10: Phase boundary in the $\left(\alpha, W_{2}\right)$-plane between a stable homogeneous state and a marginal state of a symmetric two-population IF network. Boundary curves are shown for $C=0.25$ and various values of $W_{0}$.

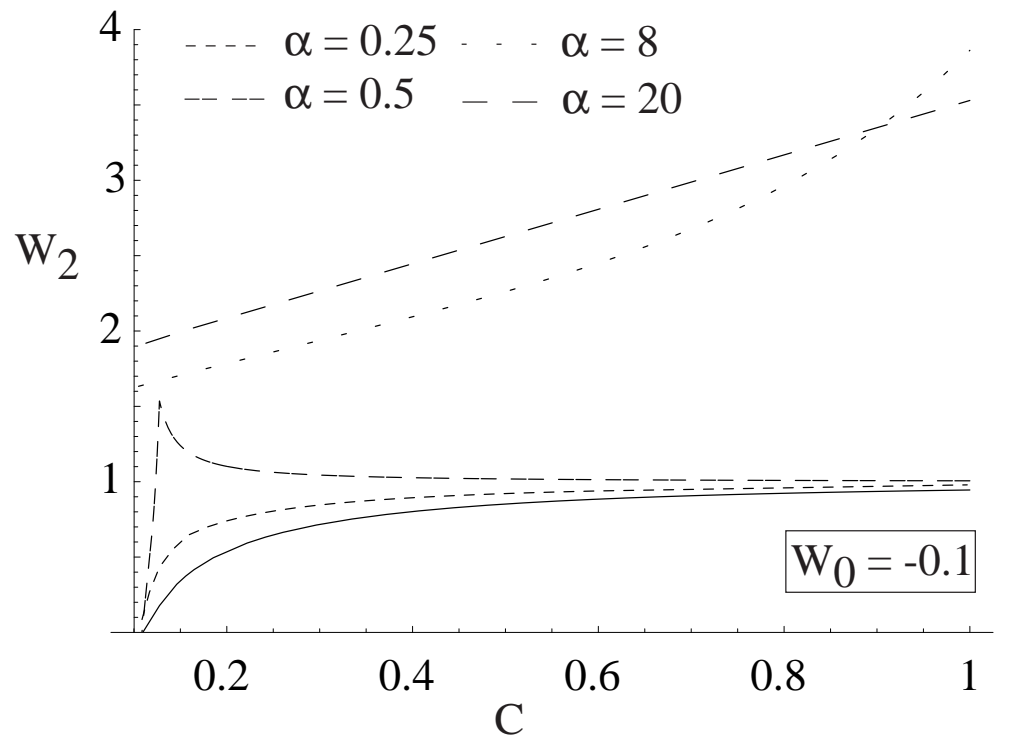

Figure 11: Phase boundary in the $\left(C, W_{2}\right)$-plane between a stable synchronous state and a marginal states of a symmetric two-population IF network. Boundary curves are shown for $W_{0}=-0.1$ and various values of the inverse rise-time $\alpha$. The solid curve corresponds to the analog model whereas the dashed curves correspond to the IF model. 
region below a given boundary curve includes the origin. Weak coupling theory shows that in a neighborhood of the origin the synchronous state is stable. Therefore, the boundary curve is a locus of bifurcation points separating a stable homogeneous state from a marginal non-phaselocked state. It should also be noted that, for each boundary curve in figures 8-10, the left-hand branch signals the onset of a (subcritical) Hopf bifurcation $\left(\omega_{0} \neq \pi\right)$ whereas the right-hand branch signals the onset of a (subcritical) period doubling bifurcation $\left(\omega_{0}=\pi\right)$; the opposite holds true in figure 11. However, there is no qualitative difference in the observed behaviour induced by these two types of instability.

Direct numerical simulations confirm that when a phase boundary curve is crossed from below, the IF network jumps to a stable marginal state consisting of a sharp orientation tuning curve as determined by the spatial distribution of the mean (time-averaged) firing rates $a(\phi)$. The latter are defined according to $a(\phi)=\bar{\Delta}(\phi)^{-1}$ where

$$
\bar{\Delta}(\phi)=\lim _{M \rightarrow \infty} \frac{1}{2 M+1} \sum_{m=-M}^{M} \Delta^{m}(\phi)
$$

with $\Delta^{m}(\phi)$ given by equation (4.3). (We do not distinguish between excitatory and inhibitory neurons here since they behave identically in a symmetric two-population network). The resulting long-term average behavior of the IF network for $W_{2}>W_{2 c}$ is illustrated in figure 12 with $W_{0}=-0.1, \alpha=0.5$ and $C=0.25$. For the given choice of parameter values, the activity profile is in good quantitative agreement with the corresponding profile obtained in the analog version of the network (see figure 4). Moreover, as in the case of the analog network, (i) the marginal state exhibits hysteresis (suggesting that the bifurcation is subcritical) and (ii) the center of the sharp orientation tuning curve is able to lock to a slowly rotating, weakly-tuned external stimulus (see figure 13).

Comparison of the phase diagrams of the IF model (figures 8-11) and the corresponding analog model (figure 3) shows that there is a major difference in the predicted value of the critical coupling $W_{2 c}$ in the two models. One finds that the critical coupling $W_{2 c}$ in the IF model converges to the corresponding analog result (which is $\alpha$-independent) in the limit $\alpha \rightarrow 0$. This is particularly clear in figure 11. However, for non-zero $\alpha$, we only expect good quantitative agreement between the two models when (i) there is an approximate balance between the mean 


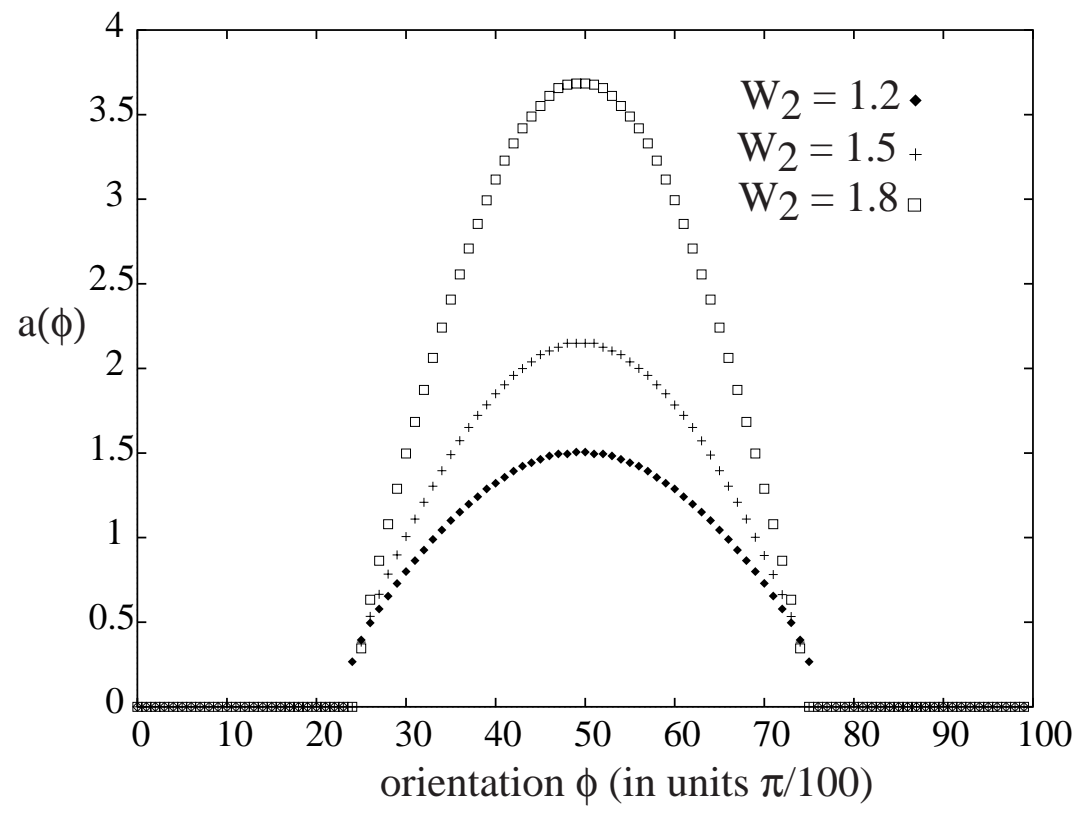

Figure 12: Sharp orientation tuning curves for a symmetric two-population IF network $(N=$ 100). The mean firing rate $a(\phi)$ is plotted as a function of orientation for $W_{2}=1.2,1.5,1.8$. Other parameter values are as in figure 4.
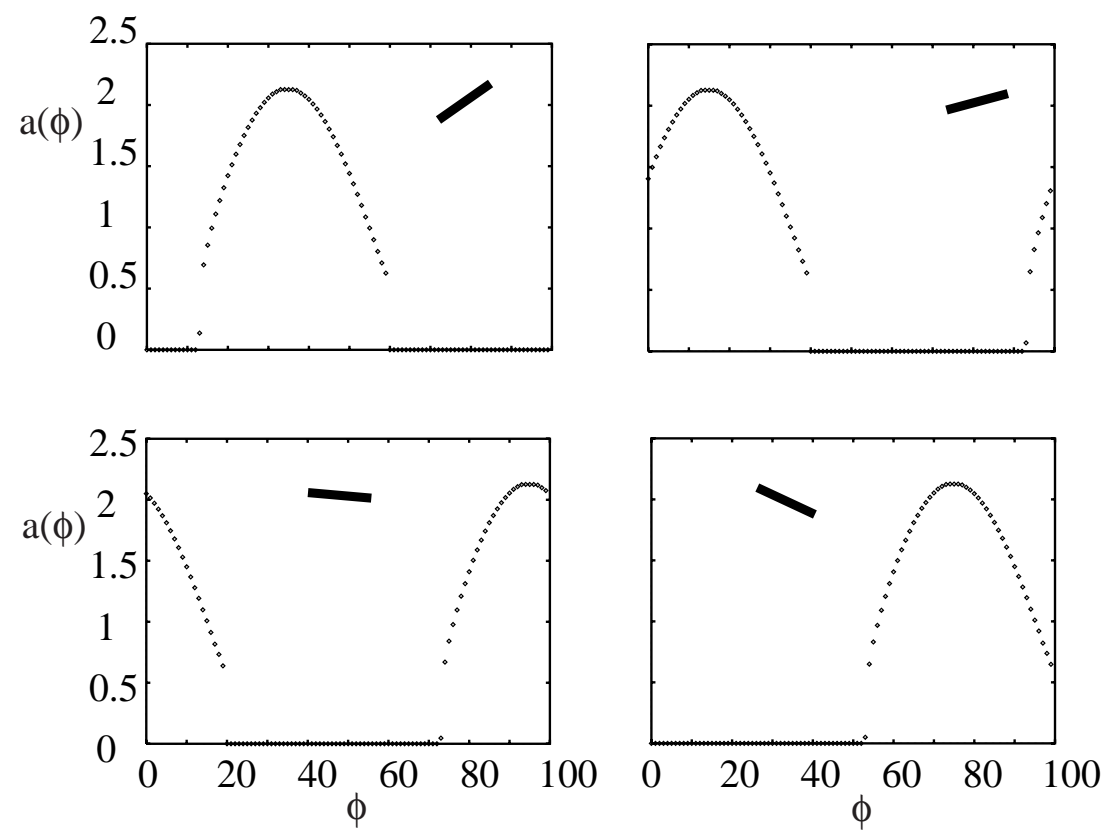

Figure 13: Orientation tuning curve in a symmetric two-population IF network $(N=100)$ locking to a slowly rotating, weakly-tuned external stimulus. Same parameter values as figure 5. 
excitatory and inhibitory coupling $\left(W_{0} \approx 0\right)$ and (ii) synaptic interactions are sufficiently slow $(\alpha \ll 1)$. Both these conditions hold in figures 12 and 13 . Outside this parameter regime, the higher value of $W_{2 c}$ for the IF model leads to relatively high levels of mean firing rates in the activity profile of the marginal state, since large values of $W_{2}$ imply strong short-range excitation. The condition $W_{0} \approx 0$ can be understood in terms of the self-consistency condition for the collective period $T$ of the synchronous state given by equation (4.4). As $W_{0}$ becomes more negative the period $T$ increases (see figure 7 ) so that $\alpha T>1$ and the reduction to the analog model is no longer a good approximation. (In Bressloff and Coombes (1999) the period $T$ is kept fixed by varying the external input).

\subsection{Quasiperiodicity and inter-spike interval variability}

We now explore in more detail the nature of the spatio-temporal dynamics of the ISIs occurring in the marginal state of the IF model. We shall proceed by fixing the parameters $W_{0}, W_{2}$ and $C$ such that a sharp orientation tuning curve exists and considering what happens as $\alpha$ is increased. In figure 14 we plot the ISI pairs $\left(\Delta^{n-1}(\phi), \Delta^{n}(\phi)\right)$, integer $n$, for all the excitatory neurons in the ring. It can be seen from figure 14(a) that for relatively slow synapses $(\alpha=2)$ the temporal fluctuations in the ISIs are negligible. There exists a set of spatially separated points reflecting the $\phi$-dependent variations in the mean firing rates $a(\phi)$ as shown in figure 12 . However, as $\alpha$ is increased, the system bifurcates to a state with periodic or quasiperiodic fluctuations of the ISIs on spatially separated invariant circles (figure 14(b)). Although the avearge behavior is still consistent with that found in the corresponding analog model, the additional fine structure associated with these orbits is not resolved by the analog model. In order to characterize the size of the ISI fluctuations we define a deterministic coefficient of variation $C_{V}(\phi)$ for a neuron $\phi$ according to

$$
C_{V}(\phi)=\frac{\sqrt{\overline{(\Delta(\phi)-\bar{\Delta}(\phi))^{2}}}}{\bar{\Delta}(\phi)}
$$

with averages defined by (4.16). The $C_{V}(\phi)$ is plotted as a function of $\phi$ for various values of $\alpha$ in figure 15. This shows that the relative size of the deterministic fluctuations in the mean firing rate is an increasing function of $\alpha$. For slow synapses $(\alpha \rightarrow 0)$, the $C_{V}$ is very small indicating 

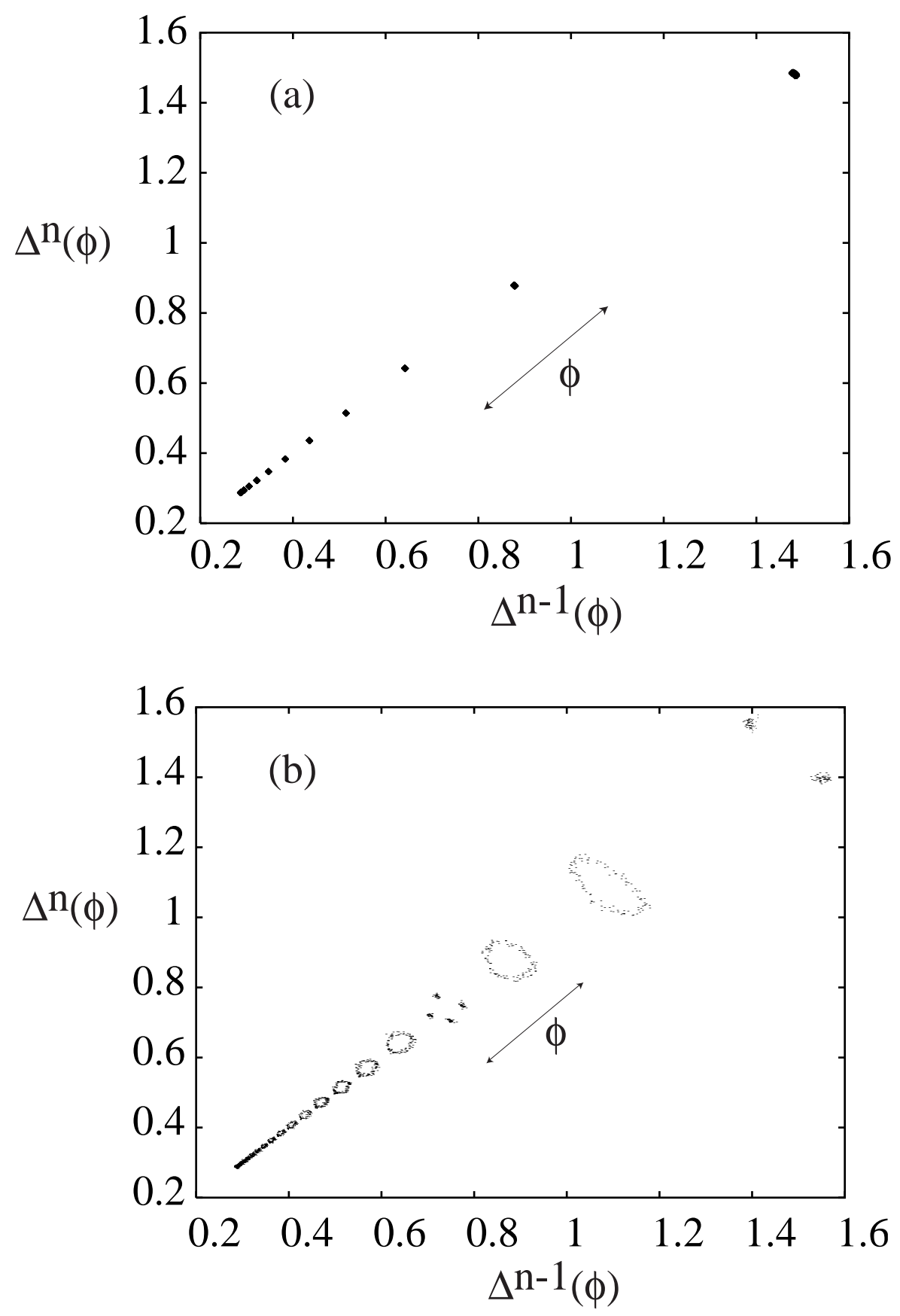

Figure 14: Separation of the inter-spike interval (ISI) orbits for a symmetric two-population IF network $(N=100)$ with $W_{0}=-0.5, W_{2}=2.0, C=0.25$. (a) $\alpha=2$. (b) $\alpha=5$. The (projected) attractors of the inter-spike interval (ISI) pairs with coordinates, $\left(\Delta^{n-1}(\phi), \Delta^{n}(\phi)\right)$ is shown for all excitatory neurons. 


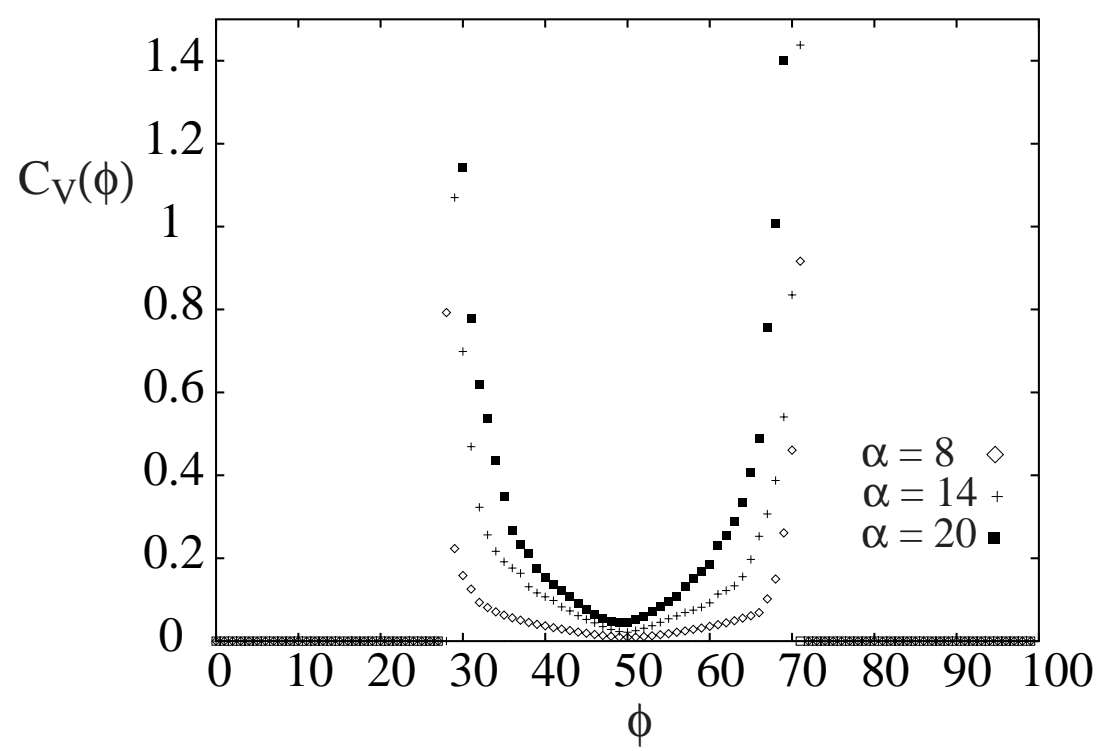

Figure 15: Plot of the coefficient of variation $C_{V}(\phi)$ as a function of $\phi$ for various values of the inverse rise-time $\alpha$. All other parameter values are as in figure 14 .

an excellent match between the IF and analog models. However, the fluctuations become much more significant when the synapses are fast. In particular, groups of neurons close to the edge of the activity profile (so that the mean firing rate is relatively small) can have a $C_{V}$ close to unity.

In figure 16 we plot $\left(\Delta^{n-1}(\phi), \Delta^{n}(\phi)\right)$ for two selected neurons, one with a low $C_{V} /$ fast firing rate and the other with a high $C_{V} /$ low firing rate. Results are shown for $\alpha=8$ and $\alpha=20$. Corresponding ISI histograms and spike trains are presented in figures 17 and 18. These figures establish that for fast synapses the ISIs display highly irregular orbits with the invariant circles of figure 14(b) no longer present. An interesting question that we hope to pursue further elsewhere concerns whether or not the underlying attractor for the ISIs supports chaotic dynamics. For it is well known that the break-up of invariant circles is one possible route to chaos as has previously been demonstrated in a number of classical fluid dynamics experiments (Bergé et al 1986). As partial evidence for chaotic ISI dynamics we plot the power spectrum $|h(p)|^{2}$ of a neuron with high $C_{V}$ in figure 19 . We consider a sequence of ISIs over $M+1$ firing events 

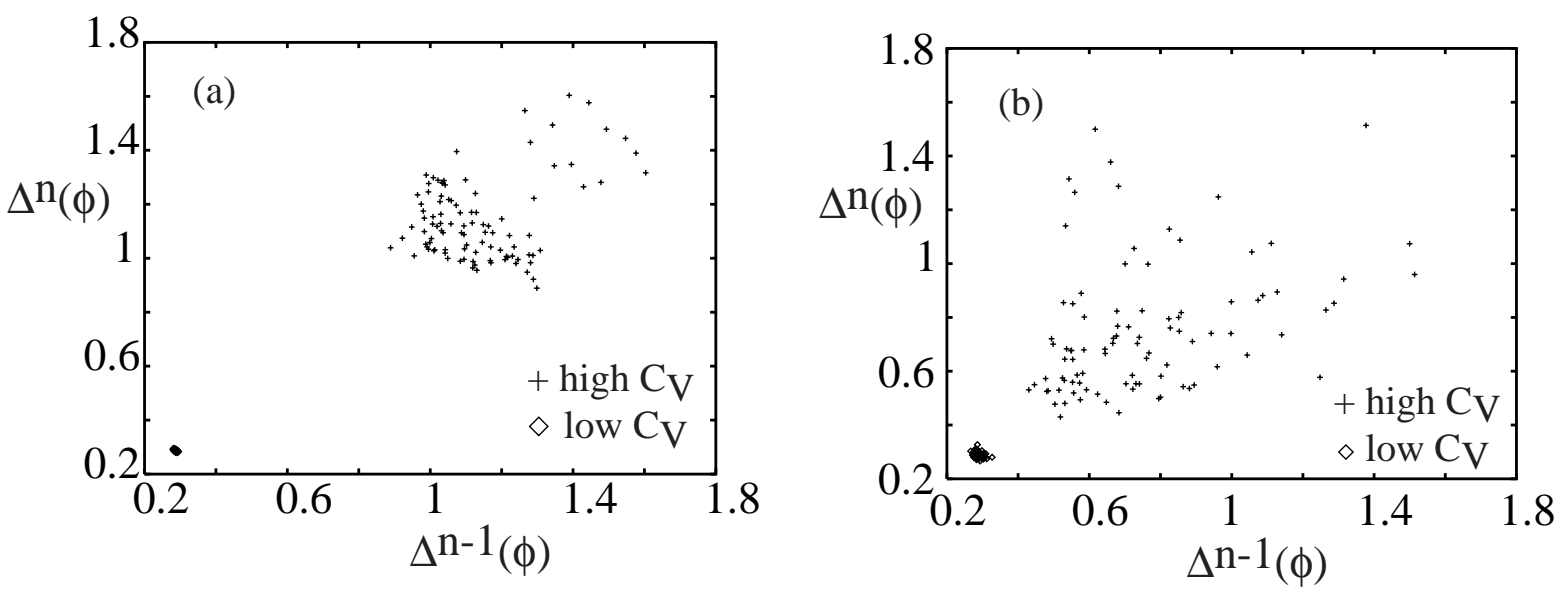

Figure 16: Same as figure 14 except that (a) $\alpha=8$ and (b) $\alpha=20$. The attractor is shown for two excitatory neurons, one with a low $C_{V}$ and the other with a high $C_{V}$.
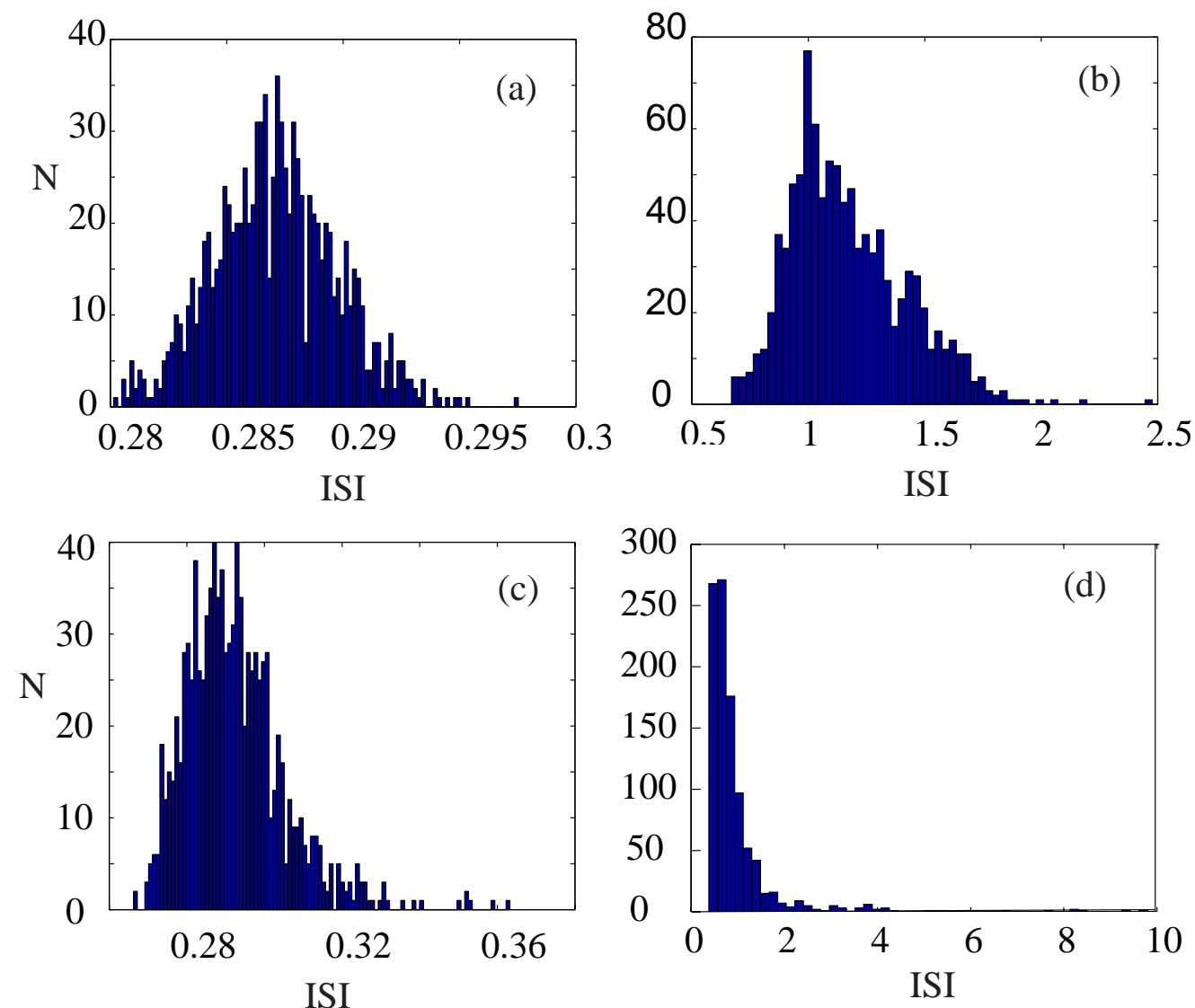

Figure 17: Histograms of 1000 firing events for selected excitatory neurons in a symmetric twopopulation IF network: (a) $\alpha=8$, low $C_{V}$ (b) $\alpha=8$, high $C_{V}$ (c) $\alpha=20$, low $C_{V}$ (d) $\alpha=20$, high $C_{V}$. All other parameter values are as in figure 14 . 

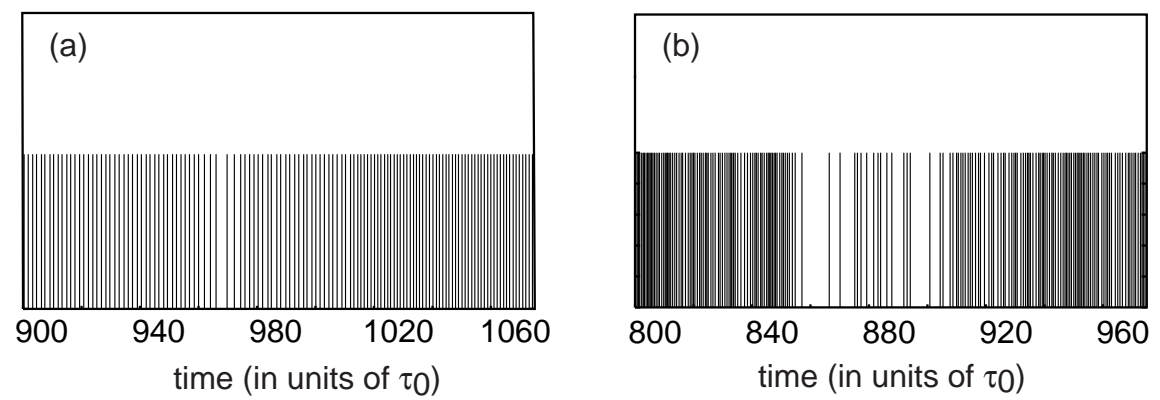

Figure 18: Spike train of an excitatory neurons in a symmetric two-population IF network: (a) $\alpha=8$, high $C_{V}$ (b) $\alpha=20$, high $C_{V}$. All other parameter values are as in figure 14 .

$\left\{\Delta_{k}, k=1, \ldots M\right\}$ and define

$$
h(p)=\frac{1}{\sqrt{M}} \sum_{k=1}^{M} \mathrm{e}^{2 \pi i k p} \Delta_{k}
$$

where $p=m / M$ for $m=1, \ldots, M$. Although the numerical data is rather noisy it does appear that there is a major difference between the quasiperiodic regime shown in figure 14 and the high- $C_{V}$ regime of figure 16. The latter possesses a broad-band spectrum, which is indicative of (but not conclusive evidence for) chaos.

There is currently considerable interest in possible mechanisms for the generation of high $C_{V} \mathrm{~s}$ in networks of cortical neurons. This follows the recent observation by Softy and Koch (1993) of high variability of the inter-spike intervals in data from cat V1 and MT neurons. Such variability is inconsistent with the standard notion that a neuron integrates over a large number of small (excitatory) inputs, since this would lead to a regular firing pattern (as a consequence of the law of large numbers). Two alternative approaches to resolving this dilemma have been proposed. The first treats cortical neurons as coincidence detectors that fire in response to a relatively small number of excitatory inputs arriving simultaneously at the (sub)millisecond level - a suggested mechanism for the amplification of a neuron's response is active dendrites (Softy and Koch 1993). The second approach retains the picture of a neuron as a synaptic integrator by incorporating a large number of inhibitory inputs to balance the effects of excitation so that a neuron operates close to threshold (Usher, Stemmler, Koch and Olami 1994; Tsodyks and Sejnowski 1995; van 

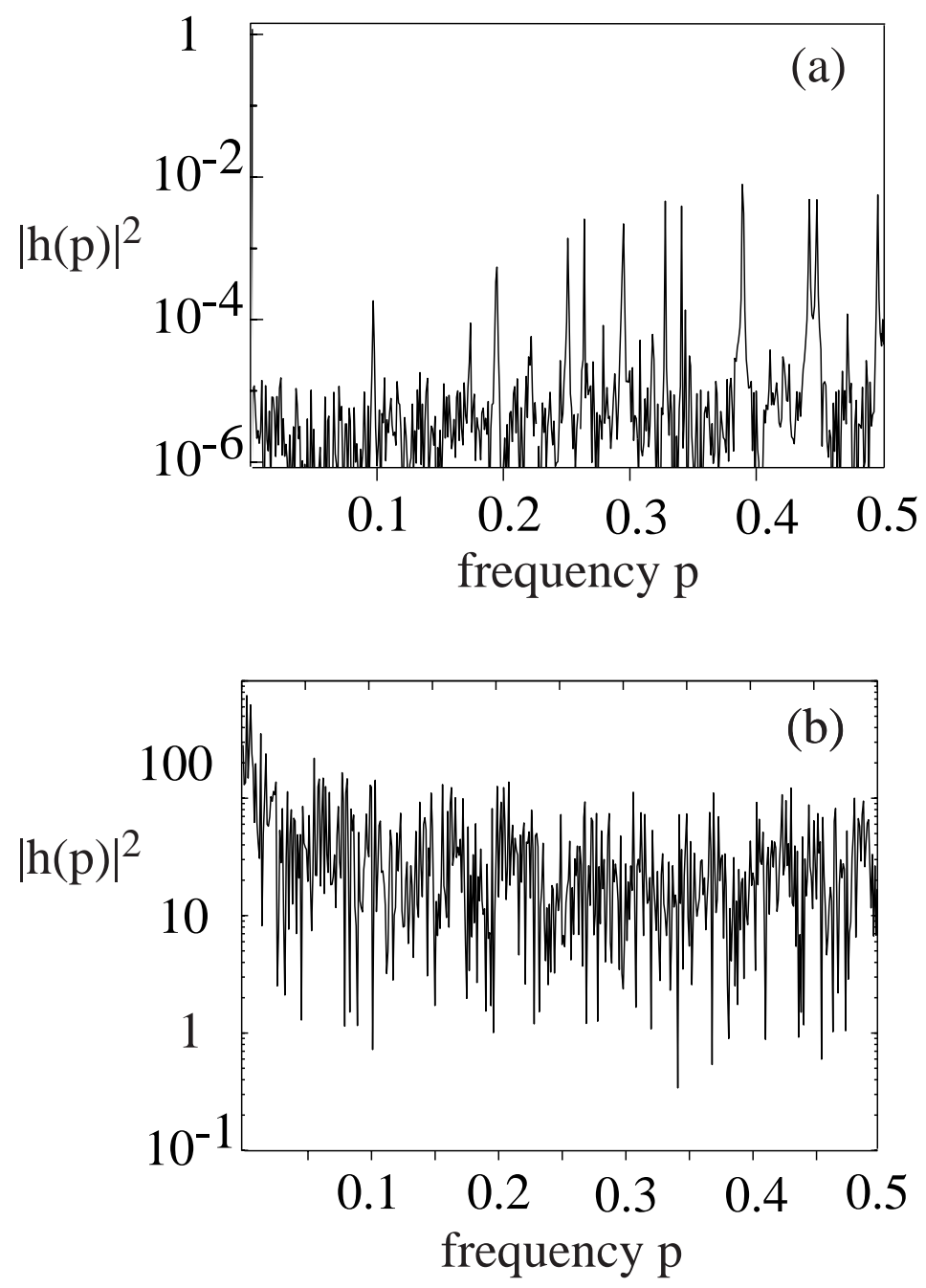

Figure 19: (a) Spectrum of a neuron in the quasiperiodic regime $(\alpha=5)$. (b) Spectrum of a high- $C_{V}$ neuron with $\alpha=20$. All other parameter values are as in figure 14 . 
Vreeswijk and Sompolinsky 1996,1998). These latter studies incorporate a degree of randomness into a network either in the form of quenched disorder in the coupling or through synaptic noise. In this paper we have shown that even an ordered network evolving deterministically can support large fluctuations in the ISIs provided that the synapses are sufficiently fast. For the activity profile shown in figure 15 , only a subset neurons have a $C_{V}$ close to unity. In order to achieve a consistently high $C_{V}$ across the whole network, it would appear necessary to have a more broadly tuned activity profile and some level of randomness (see Lin, Pawelzik, Ernst and Sejnowski 1998).

\section{$5 \quad$ Intrinsic traveling wave profiles}

So far we have restricted our analysis of orientation tuning to the case of a symmetric twopopulation model in which the excitatory and inhibitory populations behave identically. Sharp orientation tuning can still occur if the symmetry conditions (3.8) are no longer imposed, provided that the second harmonic eigenmodes are excited first when the homogeneous resting state is destabilized ( $n=2$ in equation (3.3) or (4.9)). Now, however, the heights of the excitatory and inhibitory activity profiles will generally differ. More significantly, it is possible for traveling wave profiles to occur even in the absence of a rotating external stimulus (Ben Yishai et al 1997). In order to illustrate this phenomenon, suppose that equation (3.7) is replaced by the conditions

$$
C_{L}=C, \quad \mathbf{W}_{0}=\left(\begin{array}{cc}
W_{0}^{E} & -W_{0}^{I} \\
W_{0}^{E} & -W_{0}^{I}
\end{array}\right), \quad \mathbf{W}_{2}=\left(\begin{array}{cc}
W & -K \\
K & -W
\end{array}\right)
$$

with $K>W$. First consider the analog model analyzed in section 3. Under the conditions (5.1) the fixed point equation (3.1) has solutions $\bar{X}_{L}=\bar{X}, L=E, I$, where $\bar{X}=W_{0} f(\bar{X})+C$ with $W_{0}=W_{0}^{E}-W_{0}^{I}$. Linearization about this fixed point again yields the eigenvalue equation (3.8), with the eigenvalues of the weight matrices $\mathbf{W}_{n}$ of equation (5.1) now given by

$$
\lambda_{0}^{+}=W_{0}, \quad \lambda_{0}^{-}=0, \quad \lambda_{2}^{ \pm}= \pm i W_{2}, W_{2} \equiv \sqrt{K^{2}-W^{2}}
$$

Equations (3.8) and (5.2) imply that the fixed point $\bar{X}$ is stable with respect to excitation of the $n=0$ modes provided that $\gamma W_{0}<1$. On the other hand, substituting for $\lambda_{2}^{ \pm}$using equation 
(5.2) shows that there exists solutions for $\nu$ of the form

$$
\frac{\nu}{\alpha}=-1+\sqrt{\frac{\gamma W_{2}}{2}}(1 \pm i)
$$

Hence, at a critical coupling $W_{2}=W_{2 c} \equiv 2 / \gamma$ the fixed point undergoes a Hopf bifurcation due to the occurrence of a pair of pure imaginary eigenvalues $\nu= \pm i \alpha$. The excited modes take the form $\mathbf{Z}^{ \pm} \mathrm{e}^{i(2 \phi \pm \alpha t)}$, where $\mathbf{Z}^{ \pm}$are the eigenvectors associated with $\lambda_{2}^{ \pm}$, and correspond to traveling wave activity profiles with rotation frequency $\alpha$.

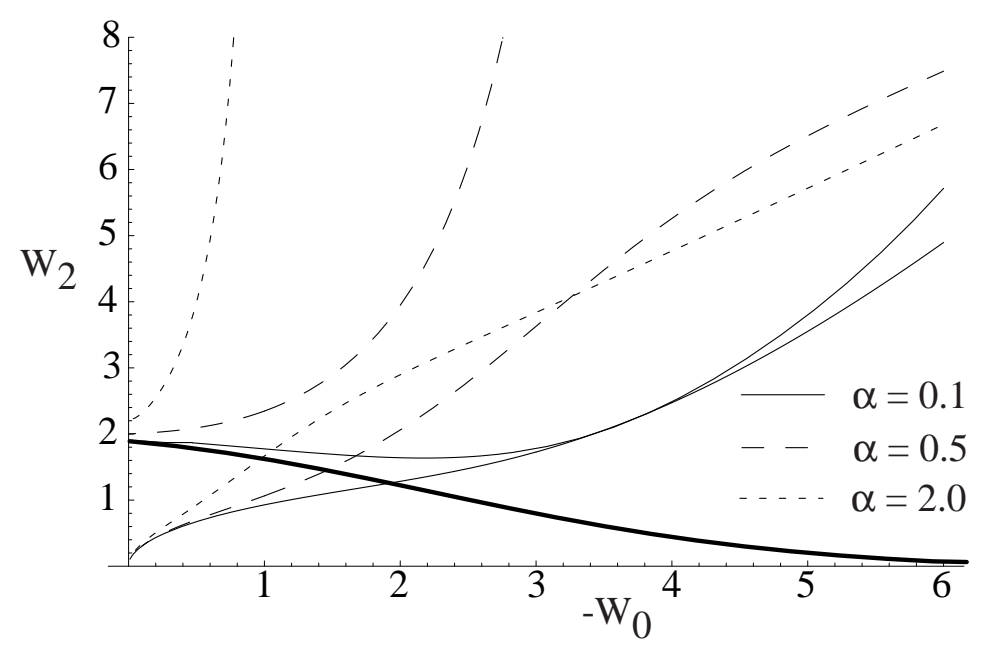

Figure 20: Phase boundary in the $\left(W_{0}, W_{2}\right)$-plane between a stable synchronous state and a traveling marginal state of an asymmetric two-population IF network. Boundary curves are shown for $C=0.25$ and various values of the inverse rise-time $\alpha$. For each $\alpha$ there are two solution branches, the lower one of which determines the point of instability of the synchronous state. The boundary curve for the analog model is also shown (thick line).

Let us now turn to the case of the IF model analyzed in section 4. Linear stability analysis of the synchronous state generates the eigenvalue equation (4.13) with $\lambda_{n}^{ \pm}$given by equation (5.2). Following similar arguments to section 3, it can be established that the synchronous state is stable in the weak coupling regime provided that $W_{0}<0$. It is also stable with respect to excitations of the $n=0$ modes for arbitrary coupling strength. Therefore, we look for strong coupling instabilities of the synchronous state by substituting $\nu=i \omega$ in equation (4.13) for 
$n=2$. Equating real and imaginary parts then leads to the pair of equations

$$
A_{T}[\cos (\omega)-1]=W_{2} \widetilde{S}(\omega)-W_{0} \widetilde{C}(0), \quad A_{T} \sin (\omega)=W_{2} \widetilde{C}(\omega)
$$

Examples of boundary curves are shown in figure 20 for $W_{2}$ as a function of $W_{0}$ with $\alpha$ fixed. For each $\alpha$ there are two solution branches, the lower one of which determines the point at which the synchronous state destabilizes. At first sight it would appear that there is a discrepancy between the IF and analog models. For the lower boundary curves of the IF model do not coincide with the phase boundary of the analog model even in the limit $\alpha \rightarrow 0$ and $W_{0} \rightarrow 0$. (Contrast figure 20 with figure 11, for example). However, numerical simulations reveal that as the lower boundary curve of the IF model is crossed from below the synchronous state destabilizes to a state consisting of two distinct synchronized populations, one excitatory and the other inhibitory. The latter state itself destabilizes for values of $W_{2}$ beyond the upper boundary curve leading to the formation of an intrinsically rotating orientation tuning curve. In figure 21 we plot the spike train of one of the IF neurons associated with such a state. The neuron clearly exhibits approximately periodic bursts at an angular frequency $\Omega \approx \pi / 6 \tau_{0}$, where the time constant $\tau_{0}$ is the fundamental unit of time. This implies that orientation selectivity can shift over a few tens of milliseconds, an effect that has recently been observed by Ringach et al (1997).

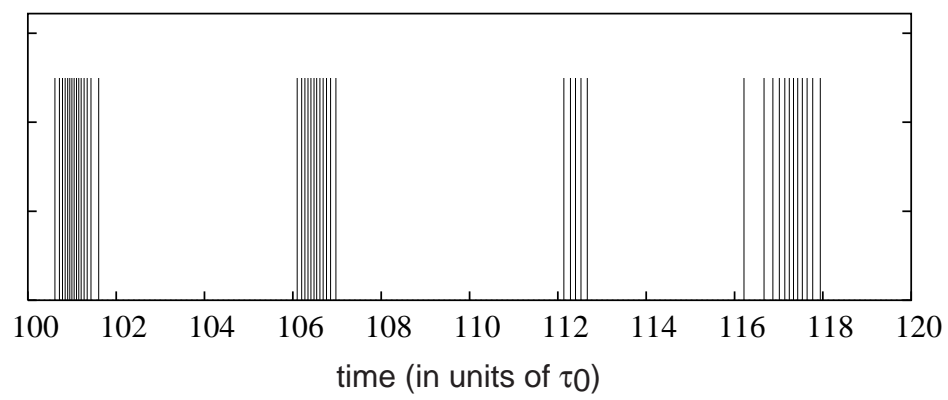

Figure 21: Oscillating spike train of an excitatory neuron in a traveling marginal state of an asymmetric two-population IF network. Here $W_{0}=-1.0, W_{2}=4.2, W_{0}^{E E}=42.5, W_{2}^{E E}=$ $20.0=J, N=50, C=1.0$ and $\alpha=2.0$. 


\section{Discussion}

Most analytical treatments of network dynamics in computational neuroscience are based on analog models in which the output of a neuron is taken to be a mean firing rate (interpreted in terms of either population or time averaging). Techniques such as linear stability analysis and bifurcation theory are used to investigate strong coupling instabilities in these networks, which induce transitions to states with complex spatio-temporal activity patterns (see Ermentrout (1998) for a review). Often a numerical comparison is made with the behavior of a corresponding network of spiking neurons based on the IF model or, perhaps, a more detailed biophysical model such as Hodgkin-Huxley. Recently, Bressloff and Coombes (1999) have develped analytical techniques for studying strong coupling instabilities in IF networks allowing a more direct comparison with analog networks to be made. In our current paper we have applied this dynamical theory of strongly coupled spiking neurons to a simple computational model of sharp orientation tuning in a cortical hypercolumn. A number of specific results were obtained that raise interesting issues for further consideration.

1. Just as in the analog model, bifurcation theory can be used to study orientation tuning in the IF model. The main results are similar in that the coefficients of the interaction function $W(\phi)=W_{0}+2 W_{2} \cos (2 \phi)$ play a key role in determining orientation tuning. If the cortical interaction is weak-both $W_{0}$ and $W_{2}$ small-then as may be expected only biases in the geniculo-cortical map can give rise to orientation tuning. However if $W_{2}$ is sufficiently large, then only a weak geniculo-cortical bias is needed to produce sharp orientation tuning at a given orientation.

2. Such a tuning is produced via a subcritical bifurcation which implies that the switching on and off of cortical cells should exhibit hysteresis (Wilson and Cowan 1972,1973). Thus both tuned and untuned states could co-exist under certain circumstances, both in the analog and the IF model. It is of interest that contrast and orientation dependent hysteresis has been observed in simple cells by Bonds (1991).

3. In addition, strong coupling instabilities in the IF model involve a discrete Hopf or period doubling bifurcation in the firing times. This typically leads to non-phase-locked behavior 
characterized by clustered but irregular variations of the ISIs. These clusters are spatially separated in phase space, which results in a localized activity pattern for the mean firing rates across the network that is modulated by fluctuations in the instantaneous firing rate. The latter can generate $C_{V}$ s of order unity. Moreover, for fast synapses the underlying attractor for the ISIs as illustrated in figure 16 appears to support chaotic dynamics. Evidence for chaos in disordered (rather than ordered) balanced networks has been presented elsewhere (van Vreeswijk and Sompolinsky 1998), although certain caution has to be taken in ascribing chaotic behaviour to potentially high-dimensional dynamical systems. Another interesting question is whether the modulations of mean firing rate observed in the responses of cat cortical neurons to visual stimuli by Gray and Singer (1989) can be explained, in part, by discrete bifurcations of the firing times in a ring of IF neurons.

4. In cases where the excitatory and inhibitory populations comprising the ring are not identical, traveling wave profiles can occur in response to a fixed stimulus. This implies that peak orientation selectivity can shift over a few tens of millisecs. Such an effect has been observed by Ringach, Hawken and Shapley (1997) and again occurs in both the analog and IF models.

5. Certain care has to be taken in identifying parameter regimes over which particular forms of network operation occur, since phase diagrams constructed for analog networks differ considerably from those of IF networks (see figure 11). For example, good quantitative agreement between the localized activity profiles of the IF and analog models only holds when (a) there is an approximate balance the mean excitatory and inhibitory coupling and (b) the synaptic interactions are sufficiently slow. 


\section{Appendix A}

Let $X(\phi, t)=\bar{X}$ be a homogeneous fixed point of the one-population model described by equation (3.9), which we rewrite in the more convenient form

$$
\frac{1}{\alpha^{2}} \frac{\partial^{2} X(\phi, t)}{\partial t^{2}}+\frac{2}{\alpha} \frac{\partial X(\phi, t)}{\partial t}+X(\phi, t)=\int_{0}^{\pi} \frac{d \phi^{\prime}}{\pi} W\left(\phi-\phi^{\prime}\right) f\left(X\left(\phi^{\prime}, t\right)\right)+C
$$

For the sake of generality, suppose that the weight distribution $W(\phi)$ is given by

$$
W(\phi)=W_{0}+2 \sum_{k=1}^{\infty} W_{k} \cos (2 k \phi)
$$

Expand the nonlinear firing-rate function about the fixed point $\bar{X}$ as in equation (3.11). Define the linear operator

$$
\hat{L} X=\frac{1}{\alpha^{2}} \frac{\partial^{2} X}{\partial t^{2}}+\frac{2}{\alpha} \frac{\partial X}{\partial t}+X-\gamma W * X
$$

in terms of the convolution $W * X(\phi)=\int_{0}^{\pi} W\left(\phi-\phi^{\prime}\right) W\left(\phi^{\prime}\right) d \phi^{\prime} / \pi$. The operator $\hat{L}$ has a discrete spectrum with eigenvalues $\nu$ satisfying (cf. equation (3.7))

$$
\left(1+\frac{\nu}{\alpha}\right)^{2}=\gamma W_{k}
$$

and corresponding eigenfunctions

$$
X(\phi, t)=z_{k} \mathrm{e}^{2 i k \phi}+z_{k}^{*} \mathrm{e}^{-2 i k \phi}
$$

Suppose that $W_{n}$ for some $n \neq 0$ is chosen as a bifurcation parameter and $\gamma_{c} W_{k}<1$ for all $k \neq n$. Then $W_{n}=W_{n, c} \equiv \gamma^{-1}$ is a bifurcation point for destabilization of the homogeneous state $\bar{X}$ due to excitation of the $n$th modes. Set $W_{n}-W_{n c}=\epsilon^{2}, W_{c}(\phi)=\left.W(\phi)\right|_{W_{n}=W_{n, c}}$, and perform the following perturbation expansion:

$$
X=\bar{X}+\epsilon X_{1}+\epsilon^{2} X_{2}+\ldots
$$

Introduce a slow time-scale $\tau=\epsilon^{2} t$ and collect terms with equal powers of $\epsilon$. This leads to a hierarchy of equations of the form

$$
\mathcal{O}(1): \quad \bar{X}=W_{0} f(\bar{X})+C
$$




$$
\begin{gathered}
\mathcal{O}(\epsilon): \quad \hat{L}_{c} X_{1}=0 \\
\mathcal{O}\left(\epsilon^{2}\right): \quad \hat{L}_{c} X_{2}=g_{2} W_{c} * X_{1}^{2} \\
\mathcal{O}\left(\epsilon^{3}\right): \quad \hat{L}_{c} X_{3}=g_{3} W_{c} * X_{1}^{3}+2 g_{2} W_{c} * X_{1} X_{2}-\left[\frac{2}{\alpha} \frac{\partial X_{1}}{\partial \tau}-\gamma f_{n} * X_{1}\right]
\end{gathered}
$$

where $\hat{L}_{c}=X-\gamma W_{c} * X$ and $f_{n}(\phi)=\cos (2 n \phi)$. The $\mathcal{O}(1)$ equation determines the fixed point $\bar{X}$. The $\mathcal{O}(\epsilon)$ equation has solutions of the form

$$
X_{1}(\phi, t)=z(\tau) \mathrm{e}^{2 i n \phi}+z^{*}(\tau) \mathrm{e}^{-2 i n \phi}
$$

We shall determine a dynamical equation for the complex amplitude $z(\tau)$ by deriving so-called solvability conditions for the higher order equations.

We proceed by taking the inner product of equations (A.9) and (A.10) with the linear eigenmode (A.11). We define the inner product of two periodic functions $U, V$ according to $\langle U \mid V\rangle=\int_{0}^{\pi} U^{*}(\phi) V(\phi) d \phi / \pi$. The $\mathcal{O}\left(\epsilon^{2}\right)$ solvability condition $\left\langle X_{1} \mid \hat{L}_{c} X_{2}\right\rangle=0$ is automatically satisfied since

$$
\left\langle X_{1} \mid W_{c} * X_{1}^{2}\right\rangle=0
$$

The $\mathcal{O}\left(\epsilon^{3}\right)$ solvability condition $\left\langle X_{1} \mid \hat{L}_{c} X_{3}\right\rangle=0$ gives

$$
\left\langle X_{1} \mid \frac{2}{\alpha} \frac{\partial X_{1}}{\partial \tau}-W_{c} * X_{1}\right\rangle=g_{3}\left\langle X_{1} \mid W_{c} * X_{1}^{3}\right\rangle+2 g_{2}\left\langle X_{1} \mid W_{c} * X_{1} X_{2}\right\rangle
$$

First, we have

$$
\left\langle\mathrm{e}^{2 i n \phi} \mid 2 \frac{\partial X_{1}}{\partial \tau}-\gamma f n * X_{1}\right\rangle=2 \frac{d z}{d \tau}-\gamma z
$$

and

$$
\left\langle\mathrm{e}^{2 i n \phi} \mid W_{c} * X_{1}^{3}\right\rangle=W_{n, c} \int_{0}^{\pi} \frac{d \phi}{\pi}\left(z \mathrm{e}^{2 i n \phi}+z^{*} \mathrm{e}^{-2 i n \phi}\right)^{3} \mathrm{e}^{-2 i n \phi}=3 W_{n, c} z|z|^{2}
$$

The next step is to determine $U_{2}$. From equation (A.9) we have

$$
\begin{aligned}
U_{2}(\phi)-\gamma \int_{0}^{\pi} \frac{d \phi^{\prime}}{\pi} W_{c}\left(\phi-\phi^{\prime}\right) U_{2}\left(\phi^{\prime}\right) & =g_{2} \int_{0}^{\pi} \frac{d \phi^{\prime}}{\pi} W_{c}\left(\phi-\phi^{\prime}\right) U_{1}\left(\phi^{\prime}\right)^{2} \\
& =g_{2}\left[z^{2} W_{2 n} \mathrm{e}^{4 i n \phi}+z^{* 2} W_{2 n} \mathrm{e}^{-4 i n \phi}+2|z|^{2} W_{0}\right](
\end{aligned}
$$


Let

$$
X_{2}(\phi)=V_{+} \mathrm{e}^{4 i n \phi}+V_{-} \mathrm{e}^{-4 i n \phi}+V_{0}+\kappa X_{1}(\phi)
$$

The constant $\kappa$ remains undetermined at this order of perturbation but does not appear in the amplitude equation for $z(\tau)$. Substituting equation (A.17) into (A.16) yields

$$
V_{+}=\frac{g_{2} z^{2} W_{2 n}}{1-\gamma W_{2 n}}, \quad V_{-}=\frac{g_{2} z^{* 2} W_{2 n}}{1-\gamma W_{2 n}}, \quad V_{0}=\frac{2 g_{2} W_{0}}{1-\gamma W_{0}}
$$

Using equation (A.18) we find that

$$
\begin{aligned}
\left\langle\mathrm{e}^{2 i n \phi} \mid W_{c} * X_{1} X_{2}\right\rangle & =W_{n, c} \int_{0}^{\pi} \frac{d \phi}{\pi}\left(z \mathrm{e}^{2 i n \phi}+z^{*} \mathrm{e}^{-2 i n \phi}\right)\left(V_{+} \mathrm{e}^{4 i n \phi}+V_{-} \mathrm{e}^{-4 i n \phi}+V_{0}+\kappa X_{1}(\phi)\right) \mathrm{e}^{-2 i n \phi} \\
& =W_{n, c}\left[z^{*} V_{+}+z V_{0}\right] \\
& =g_{2} W_{n, c} z|z|^{2}\left[\frac{W_{2 n}}{1-\gamma W_{2 n}}+\frac{2 W_{0}}{1-\gamma W_{0}}\right]
\end{aligned}
$$

Combining equations (A.14), (A.15) and (A.19) we obtain the Ginzburg-Landau equation

$$
\frac{2 \gamma}{\alpha} \frac{d z}{d \tau}=z\left(\gamma^{2}+A|z|^{2}\right)
$$

where

$$
A=3 g_{3}+2 g_{2}^{2}\left[\frac{W_{2 n}}{1-\gamma W_{2 n}}+\frac{2 W_{0}}{1-\gamma W_{0}}\right]
$$

After rescaling, $\epsilon z=Z, \tau=\epsilon^{2} t$,

$$
\frac{2 \gamma}{\alpha} \frac{d Z}{d t}=Z\left(\gamma^{2}\left[W_{n}-W_{n, c}\right]+A|Z|^{2}\right)
$$

\section{Appendix B}

In this appendix we write down explicit expressions for various quantities evaluated in the particular case of the alpha-function (2.8). First, the phase interaction function defined by equation (4.2) becomes

$$
\begin{gathered}
K_{T}(\phi)=\frac{\alpha^{2}}{1-\alpha} \frac{1-\mathrm{e}^{-T}}{1-\mathrm{e}^{-\alpha T}}\left[a_{1}(T) \mathrm{e}^{-\alpha T \phi}+T \phi \mathrm{e}^{-\alpha T \phi}+a_{2}(T) \mathrm{e}^{-T \phi}\right] \\
a_{1}(T)=\frac{T \mathrm{e}^{-\alpha T}}{1-\mathrm{e}^{-\alpha T}}-\frac{1}{1-\alpha}, \quad a_{2}(T)=\frac{1}{1-\alpha} \frac{1-\mathrm{e}^{-\alpha T}}{1-\mathrm{e}^{-T}}
\end{gathered}
$$


Second, equations (2.8) and (4.7) give

$$
\widetilde{G}_{T}(\nu)=\frac{F_{0}(T)}{1-\mathrm{e}^{-\alpha T-\nu}}-\frac{F_{1}(T) \mathrm{e}^{-\alpha T-\nu}}{\left(1-\mathrm{e}^{-\alpha T-\nu}\right)^{2}}
$$

where

$$
\begin{gathered}
F_{0}(T)=\frac{\alpha^{2} \mathrm{e}^{-T}}{(1-\alpha)^{2}}\left[\left(1-\alpha T+\alpha^{2} T\right) \mathrm{e}^{(1-\alpha) T}-1\right] \\
F_{1}(T)=\frac{T \alpha^{3} \mathrm{e}^{-T}}{1-\alpha}\left[\mathrm{e}^{(1-\alpha) T}-1\right]
\end{gathered}
$$

Finally, setting $\nu=i \omega$ in equation (B.3) and taking real and imaginary parts then leads to the result

$$
\begin{aligned}
& \widetilde{C}(\omega)=a(\omega) C(\omega)-b(\omega)\left(\cos (\omega)\left[C(\omega)^{2}-S(\omega)^{2}\right]-2 \sin (\omega) C(\omega) S(\omega)\right) \\
& \widetilde{S}(\omega)=a(\omega) S(\omega)-b(\omega)\left(\sin (\omega)\left[C(\omega)^{2}-S(\omega)^{2}\right]+2 \cos (\omega) C(\omega) S(\omega)\right)
\end{aligned}
$$

where

$$
a(\omega)=\frac{F_{0}(T)}{C(\omega)^{2}+S(\omega)^{2}}, \quad b(\omega)=\frac{F_{1}(T) \mathrm{e}^{-\alpha T}}{\left[C(\omega)^{2}+S(\omega)^{2}\right]^{2}}
$$

and $C(\omega)=1-\mathrm{e}^{-\alpha T} \cos (\omega), S(\omega)=\mathrm{e}^{-\alpha T} \sin (\omega)$. 


\section{References}

D J Amit and M V Tsodyks (1991). Quantitative study of attractor neural networks retrieving at low spike rates I: Substrate- spikes, rates and neuronal gain. Network, 2:259-274.

R Ben-Yishai, R Bar-Or Lev and H Sompolinsky (1995). Theory of orientation tuning in visual cortex. Proc. Natl. Acad. Sci. (USA), 92:3844-3848.

R Ben-Yishai, D Hansel and H Sompolinsky (1997). Traveling waves and the processing of weakly tuned inputs in a cortical network module. J. Comput. Neurosci, 4:57-77.

P. Bergé, M. Dubois and C. Vidal (1986). Order within Chaos, Wiley, New York.

A. B. Bonds (1991). Temporal dynamics of contrast gain control in single cells of the cat striate cortex. Visual Neuroscience, 6:239-255.

P C Bressloff and S Coombes (1997). Physics of the extended neuron. Int. J. Mod. Phys., 11:2343-2392.

P C Bressloff and S Coombes (1998a). Desynchronization, mode-locking and bursting in strongly coupled IF oscillators. Phys. Rev. Lett., 81: 2168-2171.

P C Bressloff and S Coombes (1998b). Spike train dynamics underlying pattern formation in integrate-and-fire oscillator networks. Phys. Rev. Lett., 81:2384-2387.

P C Bressloff and S Coombes (1999). Dynamics of strongly coupled spiking neurons. Neural Comput. In press.

B Chapman, K R Zahs and M P Stryker (1991). Relation of cortical cell orientation selectivity to alignment of receptive fields of the geniculocortical afferents that arborize within a single orientation column in ferret visual cortex. J. Neurosci., 11:1347-1358.

J D Cowan (1968). Statistical Mechanics of Nervous Nets. In E R Caianiello Ed.Neural Networks, Springer-Verlag Berlin, pp.181-188.

J D Cowan (1982). Spontaneous Symmetry Breaking in Large Scale Nervous Activity. Int. J. Quantum Chem., XXII:1059-1082. 
M C Cross and P C Hohenberg (1993). Pattern formation outside of equilibrium. Rev. Mod. Phys., 65:851-1112.

A Destexhe, Z F Mainen, and T J Sejnowski (1994). Synthesis of models for excitable membranes synaptic transmission and neuromodulation using a common kinetic formalism. J. Comp. Neurosci., 1:195-231.

R J Douglas, C Koch, M Mahowald, K Martin and H Suarez (1995). Recurrent excitation in neocortical circuits. Science, 269:981-985.

G B Ermentrout (1998). Neural networks as spatial pattern forming systems. Rep. Prog. Phys., 61:353-430.

G B Ermentrout and J D Cowan (1979a). A mathematical theory of visual hallucination pattens. Biol. Cybern., 34:137-150.

G B Ermentrout and J D Cowan (1979b). Temporal oscillations in neuronal nets. J. Math. Biol., 7:265-280.

D Ferster, S Chung and H Wheat (1997). Orientation selectivity of thalamic input to simple cells of cat visual cortex. Nature, 380:249-281.

D Ferster and C Koch (1987). Neuronal connections underlying orientation selectivity in cat visual cortex. Trends in Neurosci., 10:487-492.

A P Georgopolous (1995). Current issues in directional motor control. Trends in Neurosci., 18:506-510.

W Gerstner, J L van Hemmen, and J D Cowan (1996). What matters in neuronal locking. Neural Comput., 94:1653-1676.

C M Gray and W Singer (1989). Stimulus-specific neuronal oscillations in orientation columns of cat visual cortex. Proc. Nat. Acad. Sci., 86:1698-1702.

D Hansel and H Sompolinsky (1996). Chaos and synchrony in a model of a hypercolumn in visual cortex. J. Comput. Neurosci., 3:7-34. 
D Hansel and H Sompolinsky (1997). Modeling feature selectivity in local cortical circuits. In C Koch and I Segev eds. Methods of Neuronal Modeling 2nd edition, MIT Press Cambridge pp. $499-567$.

D H Hubel and T N Wiesel (1962). Receptive fields, binocular interaction and functional architecture in the cat's visual cortex. J. Physiol. Lond., 160:106-154.

J K Lin, K Pawelzik, U Ernst and T J Sejnowski (1998). Irregular synchronous activity in stochastically-coupled networks of integrate-and-fire neurons. Network: Comput. Neural Syst., 9:333-344.

A V Lukashin and A P Georgopolous (1994). A neural network for coding trajectories by time series of neuronal population vectors. Neural Comput., 4:57-77.

A V Lukashin and A P Georgopolous (1994). Directional operations in the motor cortex modeled by a neural network of spiking neurons. Biol. Cybern., 71:79-85.

T Mundel, A Dimitrov and J D Cowan (1997). Visual Cortex Circuitry and Orientation Tuning. Advances in Neural Information Processing Systems, 9: 887-893.

J D Murray (1990). Mathematical Biology, Springer, Berlin.

S Nelson, L Toth, B Seth and S Mur (1994). Orientation selectivity of cortical neurons during extra-cellular blockade of inhibition. Science, 265:774-777.

X Pei, T R Vidyasagar, M Volgushev and O D Creutzseldt (1994). Receptive field analysis and orientation selectivity of postsynaptic potentials of simple cells in cat visual cortex. $J$. Neurosci., 14:7130-7140.

D J Pinto, J C Brumberg, D J Simons and G B Ermentrout (1996). A quantitative population model of whisker barrels: Re-examining the Wilson-Cowan equations. J. Comput. Neurosci., 6:19-28.

R C Reid and J M Alonso (1995). Specificity of monosynaptic connections from thalamus to visual cortex. Nature, 378:281-284. 
D Ringach, M J Hawken and R Shapley (1997). Dynamics of orientation tuning in macaque primary visual cortex. Nature, 387:281-284.

A M Sillito, J A Kemp, J A Milson and N Beradi (1980). A re-evaluation of the mechanisms underlying simple cell orientation selectivity. Brain Res., 194:517-520.

W R Softky and C Koch (1993). The highly irregular firing of cortical cells is inconsistent with temporal integration of random EPSPs. J. Neurosci., 13:334-350.

D C Somers, S B Nelson and M Sur (1996). An emergent model of orientation selectivity in cat visual cortical simple cells. J. Neurosci., 15:5448-5465.

M V Tsodyks and T J Sejnowski (1995). Rapid switching in balanced cortical network models. Network: Comput. Neural Syst., 6:111-124.

M Usher, M Stemmler, C Koch, and Z Olami (1994). Network amplification of local fluctuations causes high spike rate variability, fractal firing patterns and oscillatory local field potentials. Neural Comput., 5:795-835.

C van Vreeswijk (1996). Partial synchronization in populations of pulse-coupled oscillators. Phys. Rev. E., 54:5522-5537.

C van Vreeswijk and H Sompolinsky (1996). Chaos in neuronal networks with balanced excitatory and inhibitory activity. Science, 274:1724-1726.

C van Vreeswijk and H Sompolinsky (1998). Chaotic balanced state in a model of cortical circuits. Neural Comput., 10:1321-1371.

H R Wilson and J D Cowan (1972). Excitatory and inhibitory interactions in localized populations of model neurons. Biophys. J., 12:1-24.

H R Wilson and J D Cowan (1973). A mathematical theory of the functional dynamics of cortical and thalamic nervous tissue. Kybernetik, 13:55-80.

K Zhang (1996). Representation of spatial orientation by the intrinsic dynamics of the headdirection cell ensemble: A theory. J. Neurosci, 16:2122-2126. 